FUNCTION SPACES XII

\author{
BANACH CENTER PUBLICATIONS, VOLUME 119 \\ INSTITUTE OF MATHEMATICS \\ POLISH ACADEMY OF SCIENCES \\ WARSZAWA 2019
}

\title{
LOCAL GEOMETRIC PROPERTIES IN QUASI-NORMED ORLICZ SPACES
}

\author{
ANNA KAMIŃSKA and MARIUSZ ŻYLUK \\ Department of Mathematical Sciences \\ The University of Memphis, TN 38152-3240, U.S.A. \\ E-mail:kaminska@memphis.edu,mzyluk@memphis.edu
}

\begin{abstract}
Several local geometric properties of Orlicz space $L_{\phi}$ are presented for an increasing Orlicz function $\phi$ which is not necessarily convex, and thus $L_{\phi}$ does not need to be a Banach space. In addition to monotonicity of $\phi$ it is supposed that $\phi\left(u^{1 / p}\right)$ is convex for some $p>0$ which is equivalent to that its lower Matuszewska-Orlicz index $\alpha_{\phi}>0$. Such spaces are locally bounded and are equipped with natural quasi-norms. Therefore many local geometric properties typical for Banach spaces can also be studied in those spaces. The techniques however have to be different, since duality theory cannot be applied in this case. In this article we present complete criteria, in terms of growth conditions of $\phi$, for $L_{\phi}$ to have type $0<p \leq 2$, cotype $q \geq 2$, to be (order) $p$-convex or $q$-concave, to have an upper $p$-estimate or a lower $q$-estimate, for $0<p, q<\infty$. We provide detailed proofs of most results, avoiding appealing to general not necessary theorems.
\end{abstract}

We present here a number of results on local geometric properties of Orlicz spaces $L_{\phi}$, where $\phi: \mathbb{R}_{+} \rightarrow \mathbb{R}_{+}$, called an Orlicz function, is increasing, $\phi(0)=0$, continuous and $\lim _{u \rightarrow \infty} \phi(u)=\infty$. We will assume additionally that the space $L_{\phi}$ is locally bounded, which means that topology in $L_{\phi}$ is induced by a quasi-norm. We shall consider such notions like type, cotype, (order) convexity, concavity and upper and lower estimates. In the case of Banach spaces (lattices) these properties are related, and in fact these relations have been thoroughly studied in the past decades [13. Although criteria for most of these properties have been known in the case of normed Orlicz space $L_{\phi}$ generated by a convex Orlicz function $\phi$, they have not been studied thoroughly in the case of quasi-Banach

2010 Mathematics Subject Classification: 46B20, 46E30, $47 \mathrm{~B} 38$.

Key words and phrases: quasi-norm spaces, Orlicz spaces, type, cotype, $p$-convexity, $q$-concavity, $B$-convexity.

The paper is in final form and no version of it will be published elsewhere. 
Orlicz spaces. On the other hand in paper [7] one of the authors studied Musielak-Orlicz spaces generated by the Orlicz functions (not convex) with parameter, and proved in this generality several results on (order) convexity, concavity, lower and upper estimates, though neither on type nor cotype. Theoretically from those results we can get corollaries for Orlicz spaces $L_{\phi}$ without parameters. However, both conditions on Orlicz functions with parameter and proofs in Musielak-Orlicz spaces, are far too complicated comparing with what can be done in just Orlicz spaces. In addition they cannot be interpreted instantly in the case of Orlicz functions. Therefore it is desirable to provide direct and explicit statements and their proofs of these important properties in spaces $L_{\phi}$.

The paper is divided into four sections. The first section contains preliminaries comprising of notations, definitions, some general theorems, and a number of basic results on Orlicz spaces $L_{\phi}$. The first theorem states that $\alpha_{\phi}>0$ is necessary and sufficient for the Minkowski functional to be a quasi-norm in $L_{\phi}$. It justifies a general assumption made in the entire paper that $\alpha_{\phi}>0$. We also show the relationship among Matuszewska-Orlicz indices $\alpha_{\phi}, \beta_{\phi}$ and the growth conditions $\Delta_{2}, \Delta^{p}$ and $\Delta^{* p}$. We give two regularization theorems on $\phi$, which allow us to assume that $\phi\left(u^{1 / p}\right)$ is convex or concave whenever $\phi \in \Delta^{* p}$ or $\phi \in \Delta^{p}$ respectively. It is also proved that if $\phi$ does not satisfy condition $\Delta_{2}$, then $L_{\phi}$ contains an order isomorphically isometric copy of $\ell_{\infty}$. All of these preliminary results are applied in the next sections.

Section 2 consists of two main theorems showing that for any $0<p, q<\infty, q$-convexity (resp., $p$-convexity) of $L_{\phi}$ is equivalent to lower $q$-estimate (resp., upper $p$-estimate) and this in turn is equivalent to $\phi \in \Delta^{q}$ (resp., $\phi \in \Delta^{* p}$ ). In the case of Banach lattices there exist duality among those properties [13. In that case it is possible to prove for instance the conditions on $q$-concavity, and by duality get the conditions on $p$-convexity. However in the case studied here when $L_{\phi}$ does not need to be a Banach space those theorems must be proved independently.

In Section 3 we present results on type and cotype of $L_{\phi}$. Again we are able to characterize completely these notions in terms of conditions $\Delta_{2}, \Delta^{q}$ and $\Delta^{* p}$. If $1<p \leq 2$ then $L_{\phi}$ has type $p$ if and only if $\phi \in \Delta_{2}$ and $\phi \in \Delta^{* p}$. For $0<p \leq 1$ we show that $L_{\phi}$ has type $p$ if and only if $L_{\Phi}$ is $p$-normable which in turn is equivalent to $\phi \in \Delta^{* p}$. In particular for $p=1$ we obtain that 1-normability, that is the existence of a norm equivalent to the quasi-norm in $L_{\phi}$, is equivalent to type 1 . It is not a general fact because there exist spaces with type 1 that are not normable [5, 8,

In the last Section 4 there are theorems summarizing the conditions and their relationships for type, cotype, convexity, concavity and upper and lower estimates of $L_{\phi}$ for arbitrary Orlicz function with $\alpha_{\phi}>0$. Moreover in the case when $\phi$ is convex there are stated corollaries on conditions on $B$-convexity and uniform copies in $L_{\phi}$ of finite dimensional spaces $l_{1}^{n}$ and $l_{\infty}^{n}$, closely related to nontrivial type and finite cotype, respectively.

The paper concludes with some examples. We show that given $0<p \leq 2 \leq q<\infty$ there exist Orlicz spaces having type $p$ and cotype $q$. Moreover, for any $2 \leq q<\infty$ there are Orlicz spaces with the upper index $\beta_{\phi}=q$, which do not have cotype $q$, but they have cotype $q+\epsilon$ for any $\epsilon>0$. 
All results on Orlicz spaces remain true in the case of three measure spaces, a nonatomic measure space with infinite or finite measure, or a discrete measure space identified with the set of natural numbers with counting measure. For each of these measure spaces, growth conditions of $\phi$ and indices are specified as follows. The growth conditions $\Delta_{2}$, $\Delta^{q}, \Delta^{* p}$ and indices $\alpha_{\phi}, \beta_{\phi}$ defined for all arguments, large arguments, and small arguments are corresponding to non-atomic infinite measure, non-atomic finite measure, and discrete counting measure, respectively. We will not repeat this convention while stating the results.

1. Preliminaries. Let the symbols $\mathbb{R}, \mathbb{R}_{+}$and $\mathbb{N}$ stand for reals, non-negative reals and natural numbers. Given a vector space $X$ the functional $x \mapsto\|x\|$ is called a quasi-norm if for any $x \in X$,

(1) $\|x\|=0$ if and only if $x=0$,

(2) $\|a x\|=|a|\|x\|, a \in \mathbb{R}$,

(3) there exists $C>0$ such that for all $x_{1}, x_{2} \in X$,

$$
\left\|x_{1}+x_{2}\right\| \leq C\left(\left\|x_{1}\right\|+\left\|x_{2}\right\|\right) .
$$

We will say that $(X,\|\cdot\|)$ is a quasi-Banach space if it is complete [6]. Notice that a quasi-Banach space is locally bounded [6]. For $0<p \leq 1$ the functional $x \mapsto\|x\|$ is called a $p$-norm if it satisfies the previous conditions (1) and (2) and condition

$\left(3^{\prime}\right)$ for any $x_{1}, x_{2} \in X,\left\|x_{1}+x_{2}\right\| \leq\left(\left\|x_{1}\right\|^{p}+\left\|x_{2}\right\|^{p}\right)^{1 / p}$.

A quasi-Banach space equipped with a $p$-norm is called a $p$-Banach space.

A quasi-Banach space $(X,\|\cdot\|)$ is called $p$-normable for some $0<p \leq 1$ if there exists $C>0$ such that

$$
\left\|x_{1}+\ldots+x_{n}\right\| \leq C\left(\left\|x_{1}\right\|^{p}+\ldots+\left\|x_{n}\right\|^{p}\right)^{1 / p}
$$

for all $x_{1}, \ldots, x_{n} \in X, n \in \mathbb{N}$. If $X$ is 1 -normable then we say that $X$ is normable. For any $p$-normable space $(X,\|\cdot\|)$ there exists a $p$-norm equivalent to $\|\cdot\|$. In fact

$$
\|x\|_{p}=\inf \left\{\left(\sum_{i=1}^{n}\left\|x_{i}\right\|^{p}\right)^{1 / p}: x=\sum_{i=1}^{n} x_{i}, n \in \mathbb{N}\right\}
$$

is a $p$-norm and $\|x\|_{p} \leq\|x\| \leq C\|x\|_{p}$ (see [] $)$.

TheOrem 1.1 (Aoki-Rolewicz Theorem [6, 17]). For any quasi-Banach space $(X,\|\cdot\|)$ there exist $0<p \leq 1$ and a p-norm $\|\cdot\|_{0}$ which is equivalent to $\|\cdot\|$.

REMARK 1.2. If $(X,\|\cdot\|)$ is a quasi-normed lattice, then it is easy to modify the $p$-norm above to obtain an equivalent lattice $p$-norm. Indeed, if we set $\|x\|_{1}=\inf \left\{\||y|\|_{0}\right.$ : $|x| \leq|y|\}$, the new functional $\|\cdot\|_{1}$ is a $p$-norm, which preserves the order and is equivalent to $\|\cdot\|$.

Let $r_{n}:[0,1] \rightarrow \mathbb{R}, n \in \mathbb{N}$, be Rademacher functions, that is, $r_{n}(t)=\operatorname{sign}\left(\sin 2^{n} \pi t\right)$, $t \in[0,1]$. A quasi-Banach space $X$ has type $0<p \leq 2$ if there is a constant $K>0$ such 
that, for any choice of finitely many vectors $x_{1}, \ldots, x_{n}$ from $X$,

$$
\int_{0}^{1}\left\|\sum_{k=1}^{n} r_{k}(t) x_{k}\right\| d t \leq K\left(\sum_{k=1}^{n}\left\|x_{k}\right\|^{p}\right)^{1 / p},
$$

and it has cotype $q \geq 2$ if there is a constant $K>0$ such that for any finitely many elements $x_{1}, \ldots, x_{n}$ from $X$,

$$
\left(\sum_{k=1}^{n}\left\|x_{k}\right\|^{q}\right)^{1 / q} \leq K \int_{0}^{1}\left\|\sum_{k=1}^{n} r_{k}(t) x_{k}\right\| d t .
$$

Clearly if a quasi-Banach space has type $0<p \leq 2$, respectively cotype $q \geq 2$, then it has type $r$ for any $0<r<p$, respectively cotype $r>q$. For these notions we refer to [13] for Banach spaces and to [4] for quasi-Banach spaces.

The following result by Kalton gives a connection between type $1<p \leq 2$ and 1-normability of quasi-Banach spaces.

Theorem 1.3 ([4, Theorem 4.1]). Let $1<p \leq 2$. If a quasi-Banach space has type $p$ then it is normable.

A quasi-Banach space $(X,\|\cdot\|)$ which in addition is a vector lattice and $\|x\| \leq\|y\|$ whenever $|x| \leq|y|$ is called a quasi-Banach lattice. A quasi-Banach lattice $X=(X,\|\cdot\|)$ is said to be (order) $p$-convex, $0<p<\infty$, respectively (order) $q$-concave, $0<q<\infty$, if there are positive constants $C_{p}$, respectively $D_{q}$, such that

$$
\left\|\left(\sum_{i=1}^{n}\left|x_{i}\right|^{p}\right)^{1 / p}\right\| \leq C_{p}\left(\sum_{i=1}^{n}\left\|x_{i}\right\|^{p}\right)^{1 / p}
$$

respectively,

$$
\left(\sum_{i=1}^{n}\left\|x_{i}\right\|^{q}\right)^{1 / q} \leq D_{q}\left\|\left(\sum_{i=1}^{n}\left|x_{i}\right|^{q}\right)^{1 / q}\right\|
$$

for every choice of vectors $x_{1}, \ldots, x_{n} \in X$. When referring to these notions we skip the word "order", and simply say $p$-convex or $q$-concave. We also say that $X$ satisfies an upper $p$-estimate, $0<p<\infty$, respectively a lower $q$-estimate, $0<q<\infty$, if the inequalities in the definition of $p$-convexity, respectively $q$-concavity, hold only for any choice of disjointly supported elements $x_{1}, \ldots, x_{n}$ in $X$ [4, 13. It is well known that if a quasi-Banach space is $p$-convex or has an upper $p$-convexity, then for any $0<r \leq p, X$ is $r$-convex or has an upper $r$-convexity respectively. For $q$-concavity or lower $q$-estimate the similar property holds in reverse direction 13 .

The next two results on relationships among type, cotype, convexity and concavity are true in Banach lattices.

TheOrem 1.4 ([13, Theorem 1.f.18]). A Banach lattice $X$ has type $p>1$ if and only if its dual space $X^{*}$ has cotype $p^{\prime}, 1 / p+1 / p^{\prime}=1$, and has a lower $q$-estimate for some $q<\infty$.

Theorem 1.5 ([13, Corollary 1.f.13]). A Banach lattice which has type $p>1$, is $q$-concave for some $q<\infty$. 
The next result was proved in [13, Theorem 1.d.6] for Banach lattices only, while it is shown here for quasi-Banach lattices.

Theorem 1.6. Let $(X,\|\cdot\|)$ be a quasi-Banach lattice. If $X$ is $q$-concave for some $q<\infty$, then there exists $C>0$ such that for every $x_{1}, \ldots, x_{n} \in X, n \in \mathbb{N}$, we have

$$
\int_{0}^{1}\left\|\sum_{i=1}^{n} r_{i}(t) x_{i}\right\| d t \leq C\left\|\left(\sum_{i=1}^{n}\left|x_{i}\right|^{2}\right)^{1 / 2}\right\| .
$$

If $X$ is p-convex for some $p>0$, then there exists $C>0$ such that for every $x_{1}, \ldots, x_{n}$ in $X, n \in \mathbb{N}$, we have

$$
\int_{0}^{1}\left\|\sum_{i=1}^{n} r_{i}(t) x_{i}\right\| d t \geq C\left\|\left(\sum_{i=1}^{n}\left|x_{i}\right|^{2}\right)^{1 / 2}\right\| .
$$

Proof. Recall first well known Khintchine's inequality [2, 12]. For every $1 \leq r<\infty$ there exist positive constants $A_{r}$ and $B_{r}$ such that

$$
A_{r}\left(\sum_{i=1}^{n}\left|a_{i}\right|^{2}\right)^{1 / 2} \leq\left(\int_{0}^{1}\left|\sum_{i=1}^{n} a_{i} r_{i}(t)\right|^{r} d t\right)^{1 / r} \leq B_{r}\left(\sum_{i=1}^{n}\left|a_{i}\right|^{2}\right)^{1 / 2}
$$

for every choice of scalars $a_{1}, \ldots, a_{n}$. We can assume $0<p<1<q$. Let $x_{1}, \ldots, x_{n} \in X$. Then by $q$-concavity of $X$, Khintchine's inequality and [13, Theorem 1.d.1],

$$
\begin{gathered}
\int_{0}^{1}\left\|\sum_{i=1}^{n} r_{i}(t) x_{i}\right\| d t \leq\left(\int_{0}^{1}\left\|\sum_{i=1}^{n} r_{i}(t) x_{i}\right\|^{q} d t\right)^{1 / q}=\left(\frac{1}{2^{n}} \sum_{\theta_{j}= \pm 1}\left\|\sum_{j=1}^{n} \theta_{j} x_{j}\right\|^{q}\right)^{1 / q} \\
=\left(\sum_{\theta_{j}= \pm 1}\left\|\frac{1}{2^{n / q}} \sum_{j=1}^{n} \theta_{j} x_{j}\right\|^{q}\right)^{1 / q} \leq D_{q}\left\|\left(\sum_{\theta_{j}= \pm 1}\left|\frac{1}{2^{n / q}} \sum_{j=1}^{n} \theta_{j} x_{j}\right|^{q}\right)^{1 / q}\right\| \\
=D_{q}\left\|\left(\frac{1}{2^{n}} \sum_{\theta_{j}= \pm 1}\left|\sum_{j=1}^{n} \theta_{j} x_{j}\right|^{q}\right)^{1 / q}\right\|=D_{q}\left\|\left(\int_{0}^{1}\left|\sum_{i=1}^{n} r_{i}(t) x_{i}\right|^{q} d t\right)^{1 / q}\right\| \\
\leq D_{q} B_{q}\left\|\left(\sum_{i=1}^{n}\left|x_{i}\right|^{2}\right)^{1 / 2}\right\| .
\end{gathered}
$$

On the other hand, applying $p$-convexity of $X$ and Khintchine's inequality,

$$
\begin{aligned}
\int_{0}^{1}\left\|\sum_{i=1}^{n} r_{i}(t) x_{i}\right\| d t & \geq\left(\int_{0}^{1}\left\|\sum_{i=1}^{n} r_{i}(t) x_{i}\right\|^{p} d t\right)^{1 / p}=\left(\sum_{\theta_{j}= \pm 1}\left\|\frac{1}{2^{n / p}} \sum_{j=1}^{n} \theta_{j} x_{j}\right\|^{p}\right)^{1 / p} \\
& \geq C_{p}\left\|\left(\sum_{\theta_{j}= \pm 1} \frac{1}{2^{n}}\left|\sum_{j=1}^{n} \theta_{j} x_{j}\right|^{p}\right)^{1 / p}\right\|=C_{p}\left\|\left(\int_{0}^{1}\left|\sum_{i=1}^{n} r_{i}(t) x_{i}\right|^{p} d t\right)^{1 / p}\right\| \\
& \geq C_{p} A_{p}\left\|\left(\sum_{i=1}^{n}\left|x_{i}\right|^{2}\right)^{1 / 2}\right\| .
\end{aligned}
$$

In the sequel $(\Omega, \Sigma, \mu)$ denotes a $\sigma$-finite measure space. The space of all (equivalence classes of) $\Sigma$-measurable real functions defined on $\Omega$ is denoted by $L^{0}=L^{0}(\mu)$. $L^{0}$ is a lattice with the pointwise order, that is $f \leq g$ whenever $f(t) \leq g(t)$ a.e. 
In this article we will consider three types of measure spaces. $(\Omega, \Sigma, \mu)$ will be either non-atomic with $\mu(\Omega)=\infty$ or non-atomic with $\mu(\Omega)<\infty$, or purely atomic with counting measure. In the last case we identify $\Omega$ with $\mathbb{N}$, where $\Sigma$ consists of all subsets of $\mathbb{N}$ and $\mu(\{n\})=1$ for every $n \in \mathbb{N}$.

A function $\phi: \mathbb{R}_{+} \rightarrow \mathbb{R}_{+}$is said to be an Orlicz function if $\phi(0)=0, \phi$ is increasing (meaning strictly increasing), continuous, and $\lim _{u \rightarrow \infty} \phi(u)=\infty$.

Several growth conditions of Orlicz functions will be considered. These conditions and also equivalence relations between Orlicz functions will be given in three different versions dependently on the measure space. Further, without additional comments (unless they are necessary), we will always associate the infinite non-atomic measure with conditions "for all arguments", the finite non-atomic measure with properties "for large arguments" and finally the purely atomic measure with those defined "for small arguments".

In the sequel we will use the numbers called Matuszewska-Orlicz indices, which characterize growth conditions of real valued functions, in particular Orlicz functions $\phi$. There are three parallel definitions, for all, large or small arguments.

Definition 1.7. Let $\phi$ be an Orlicz function. The lower Matuszewska-Orlicz index $\alpha_{\phi}$ for all arguments (resp. large arguments; small arguments) is defined as follows

$$
\begin{gathered}
\alpha_{\phi}=\sup \{p \in \mathbb{R}: \exists c>0 \text { (resp. } \exists c>0, v \geq 0 ; \exists c>0, v>0 \text { ) such that } \\
\phi(a u) \geq c a^{p} \phi(u)
\end{gathered}
$$

for all $a \geq 1$ and $u \geq 0$ (resp. $u \geq v ; 0<u \leq a u \leq v)\}$.

We define upper Matuszewska-Orlicz index $\beta_{\phi}$ for all arguments (resp. large arguments; small arguments) as

$$
\begin{gathered}
\beta_{\phi}=\inf \{p \in \mathbb{R}: \exists c>0 \text { (resp. } \exists c>0, v \geq 0 ; \exists c>0, v>0 \text { ) such that } \\
\qquad \phi(a u) \leq c a^{p} \phi(u) \\
\text { for all } a \geq 1 \text { and } u \geq 0 \text { (resp. } u \geq v ; 0<u \leq a u \leq v)\} .
\end{gathered}
$$

The Orlicz space generated by an Orlicz function $\phi$ is denoted by $L_{\phi}$ and is defined as the set of all $f \in L^{0}$ such that

$$
I_{\phi}(\lambda f)=\int_{\Omega} \phi(\lambda|f(t)|) d \mu=\int \phi(\lambda|f|)<\infty
$$

for some $\lambda>0$ dependent on $f$. The Minkowski functional of the set $\left\{f \in L^{0}: I_{\phi}(f) \leq 1\right\}$,

$$
\|f\|=\|f\|_{\phi}=\inf \left\{\varepsilon>0: I_{\phi}(f / \varepsilon) \leq 1\right\}
$$

is finite on $L_{\phi}$. In the case of counting measure, usually the Orlicz space is denoted by $l_{\phi}$ and its elements are sequences $x=\{x(n)\}$.

The next result provides necessary and sufficient condition for $\|\cdot\|$ to be a quasinorm [15].

THEOREM 1.8. The Minkowski functional $\|\cdot\|$ is a quasi-norm in $L_{\phi}$ if and only if the upper index $\alpha_{\phi}>0$.

If $\phi$ is a convex function then $\|\cdot\|$ is a norm in $L_{\phi}$, called the Luxemburg norm. 
Proof. We will consider here only the case when the measure is non-atomic and infinite. Then according to our convention we use the index $\alpha_{\phi}$ for all arguments.

It is clear that $\|a f\|=|a|\|f\|$ for $a \in \mathbb{R}$, and $\|f\|=0$ if and only if $f=0$ a.e. If $\alpha_{\phi}>0$ then there exists $p>0$, such that $\phi(a u) \geq C a^{p} \phi(u)$ for some $0<C<1$ and all $a \geq 1, u \geq 0$. Setting then $a>1$ such that $C^{-1} a^{-p}=\frac{1}{2}$ and $K=a$ we get

$$
\phi\left(\frac{u}{K}\right) \leq \frac{1}{2} \phi(u), \quad \text { for all } u \geq 0 .
$$

Observe also that in view of monotonicity of $\phi$ we have

$$
\phi(\lambda t+(1-\lambda) s) \leq \phi(t)+\phi(s), \quad \text { for all } t, s \geq 0,0 \leq \lambda \leq 1 .
$$

Letting then $f, g \in L_{\phi}$ with $I_{\phi}\left(\frac{f}{\alpha}\right) \leq 1$ and $I_{\phi}\left(\frac{g}{\beta}\right) \leq 1$ we get

$$
\begin{aligned}
I_{\phi}\left(\frac{f+g}{K(\alpha+\beta)}\right) & \leq \int \phi\left(\frac{\alpha}{\alpha+\beta} \frac{|f|}{K \alpha}+\frac{\beta}{\alpha+\beta} \frac{|g|}{K \beta}\right) \\
& \leq I_{\phi}\left(\frac{f}{K \alpha}\right)+I_{\phi}\left(\frac{g}{K \beta}\right) \leq \frac{1}{2} I_{\phi}\left(\frac{f}{\alpha}\right)+\frac{1}{2} I_{\phi}\left(\frac{g}{\beta}\right) \leq 1
\end{aligned}
$$

It follows $\|f+g\| \leq K(\|f\|+\|g\|)$ for any $f, g \in L_{\phi}$.

If $\phi$ is convex then

$$
\phi(\lambda t+(1-\lambda) s) \leq \lambda \phi(t)+(1-\lambda) \phi(s), \text { for all } t, s \geq 0,0 \leq \lambda \leq 1 .
$$

Then analogously as in general case letting $I_{\phi}\left(\frac{f}{\alpha}\right) \leq 1$ and $I_{\phi}\left(\frac{g}{\beta}\right) \leq 1$, and applying convexity of $\phi$, we get

$$
I_{\phi}\left(\frac{f+g}{\alpha+\beta}\right) \leq \frac{\alpha}{\alpha+\beta} I_{\phi}\left(\frac{f}{\alpha}\right)+\frac{\beta}{\alpha+\beta} I_{\phi}\left(\frac{g}{\beta}\right) \leq 1,
$$

which implies $\|f+g\| \leq\|f\|+\|g\|$.

Let now $\|\cdot\|$ be a quasi-norm. Given a set $A$ with finite measure, clearly

$$
\left\|\chi_{A}\right\|=\frac{1}{\phi^{-1}(1 / \mu(A))} \text {. }
$$

By non-atomicity of $\mu$ for any $t>0$ there exist disjoint sets $A, B$ such that $\mu(A)=$ $\mu(B)=t$. By the assumption that $\|\cdot\|$ is a quasi-norm, there exists $M>1$ such that

$$
\frac{\left\|\chi_{A}+\chi_{B}\right\|}{\left\|\chi_{A}\right\|+\left\|\chi_{B}\right\|}=\frac{\phi^{-1}(1 / t)}{2 \phi^{-1}(1 /(2 t))} \leq M,
$$

for all $t>0$. Therefore setting $K=2 M$ we get

$$
2 \phi(t) \leq \phi(K t),
$$

for all $t>0$. If we now take $a \geq 1$ there exists $m \in \mathbb{N}$ such that $K^{m-1} \leq a<K^{m}$. For $p=\frac{\ln 2}{\ln K}$ we have $2^{m-1}=\left(K^{m-1}\right)^{p}$. It follows that for all $t \geq 0$ and $a \geq 1$,

$$
\phi(a t) \geq \phi\left(K^{m-1} t\right) \geq 2^{m-1} \phi(t)=\left(K^{m-1}\right)^{p} \phi(t) \geq a^{p} K^{-p} \phi(t) .
$$

Consequently $\alpha_{\phi}>0$, and the proof is completed.

For the entire paper we assume that $\alpha_{\phi}>0$. Then the space $L_{\phi}$ equipped with the Minkowski functional $\|\cdot\|$ is a quasi-Banach space. 
Recall that Orlicz functions $\phi$ and $\psi$ are equivalent for all arguments (resp. large arguments; small arguments) if there exist positive constants $K_{1}, K_{2}$ (resp. positive constants $K_{1}, K_{2}$ and $v \geq 0$; positive constants $K_{1}, K_{2}$ and $v>0$ ) such that

$$
K_{1}^{-1} \psi\left(K_{2}^{-1} u\right) \leq \phi(u) \leq K_{1} \psi\left(K_{2} u\right)
$$

for all $u \geq 0$ (resp. $u \geq v ; u \leq v$ ). Two equivalent Orlicz functions define the same Orlicz spaces with equivalent quasi-norms. In fact it is known more, two Orlicz spaces on non-atomic infinite measure (resp. non-atomic finite measure; purely atomic measure) are equal with equivalent quasi-norms if and only if the corresponding Orlicz functions are equivalent for all arguments (resp. large arguments; small arguments) [15, 16, 14, .

We start with the most classical growth property of $\phi, \Delta_{2}$-condition. We say that an Orlicz function $\phi$ satisfies condition $\Delta_{2}\left(\phi \in \Delta_{2}\right)$ for all arguments (resp. large arguments; small arguments) if there exists a positive constant $K$ (resp. there exist a positive constant $K$ and $v \geq 0$; a positive constant $K$ and $v>0$ ) such that

$$
\phi(2 u) \leq K \phi(u)
$$

for all $u \geq 0$ (resp. $u \geq v ; u \leq v)$.

The condition $\Delta_{2}$ is intimately connected to more subtle conditions $\Delta^{p}$ and $\Delta^{* p}$.

Definition 1.9. An Orlicz function $\phi$ satisfies condition $\Delta^{q}\left(\phi \in \Delta^{q}\right), q>0$, for all arguments (resp. large arguments; small arguments) if there exists a positive constant $K$ (resp. there exist a positive constant $K$ and $v \geq 0$; a positive constant $K$ and $v>0$ ) such that

$$
\phi(a u) \leq K a^{q} \phi(u)
$$

for all $a \geq 1$ and $u \geq 0$ (resp. $u \geq v ; a u \leq v$ ).

An Orlicz function $\phi$ satisfies condition $\Delta^{* p}\left(\phi \in \Delta^{* p}\right), p>0$, for all arguments (resp. large arguments; small arguments) if there exists a positive constant $K$ (resp. there exist a positive constant $K$ and $v \geq 0$; a positive constant $K$ and $v>0$ ) such that

$$
\phi(a u) \geq K a^{p} \phi(u)
$$

for all $a \geq 1$ and $u \geq 0$ (resp. $u \geq v ; a u \leq v$ ).

REMARK 1.10. If $\phi \in \Delta^{q}$, respectively $\phi \in \Delta^{* p}$, then $\phi \in \Delta^{q_{1}}$, respectively $\phi \in \Delta^{* p_{1}}$, for any $q_{1}>q$, respectively for any $0<p<p_{1}$. Therefore

$$
\alpha_{\phi}=\sup \left\{p: \phi \in \Delta^{* p}\right\}, \quad \beta_{\phi}=\inf \left\{q: \phi \in \Delta^{q}\right\} .
$$

Given an Orlicz function $\phi$, the function $\phi^{*}$ is defined for $u \geq 0$, as

$$
\phi^{*}(u)=\sup _{w \geq 0}\{u w-\phi(w)\} .
$$

It is called the Young conjugate or the complementary function, or the Legendre transformation of $\phi$. Let us point out here that $\phi^{*}$ may assume infinite values although $\phi$ itself is finite. It is easy to check that $\phi^{*}: \mathbb{R}_{+} \rightarrow[0,+\infty]$ is convex, $\phi^{*}(0)=0, \phi^{*}$ is left continuous and $\phi^{*}$ is not identically equal to infinity. Moreover, it is well known and easy to check that $\phi^{* *} \leq \phi$ and $\phi^{* *}$ is the largest convex minorant of $\phi$. Consequently $\phi=\phi^{* *}$ whenever $\phi$ is convex [3, Theorem 1 on p. 175]. 
Equivalent relations for Orlicz functions possibly assuming infinite values (like conjugate functions) are defined in the same manner like for the Orlicz finite valued functions. Observe that in the case when $\phi^{*}$ satisfies condition $\Delta_{2}$ for all and large arguments, it assumes only finite values, and when this condition is satisfied for small arguments, then $\phi^{*}$ may be replaced by an equivalent Orlicz convex finite valued function.

Let

$$
E_{\phi}=\left\{f \in L^{0}: I_{\phi}(\lambda f)<\infty \text { for every } \lambda>0\right\} .
$$

$E_{\phi}$ is a closed subspace of $L_{\phi}$ and is usually called the subspace of finite elements. It is well known that $E_{\phi}$ is a closure in $L_{\phi}$ of the set of simple functions with finite measure supports. Moreover $L_{\phi}=E_{\phi}$ if and only if $\phi$ satisfies condition $\Delta_{2}$ [16. Theorem 8.14].

If $\phi$ is a convex Orlicz function satisfying condition $\Delta_{2}$ then the dual space $\left(E_{\phi}\right)^{*}$ is canonically isomorphic to $L_{\phi^{*}}$ via integral functionals. In fact

$$
\left(E_{\phi},\|\cdot\|_{\phi}\right)^{*} \simeq\left(L_{\phi^{*}},\|\cdot\|_{\phi^{*}}^{0}\right),
$$

where the symbol $\simeq$ means the spaces are linearly isometric. Here

$$
\|f\|_{\phi}^{0}=\|f\|^{0}=\sup \left\{\int f g: I_{\phi^{*}}(g) \leq 1\right\}
$$

is the Orlicz norm in $L_{\phi}$. It is well known that $\|f\| \leq\|f\|^{0} \leq 2\|f\|$ for $f \in L_{\phi}$ [11, 14, 16].

The next result was proved for Musielak-Orlicz spaces in [7].

Proposition 1.11. If an Orlicz function $\phi$ does not satisfy the corresponding condition $\Delta_{2}$, then $L_{\phi}$ contains an order isomorphically isometric copy of $\ell_{\infty}$.

Proof. We shall conduct the proof only in the case of a non-atomic and infinite measure $\mu$.

Our first observation is that $\phi \in \Delta_{2}$ if and only if for every $a \geq 1$ there exists $K_{a}>0$ such that

$$
\phi(a u) \leq K_{a} \phi(u)
$$

for all $u>0$. In fact for $a>1$ there exists $m \in \mathbb{N}$ such that $2^{m-1} \leq a<2^{m}$. Setting $q=\ln K / \ln 2$ and $K_{a}=2^{q} a^{q}$, for every $u \geq 0$,

$$
\phi(a u) \leq \phi\left(2^{m} u\right) \leq K^{m} \phi(u)=\left(2^{m}\right)^{q} \phi(u) \leq K_{a} \phi(u) .
$$

We claim now that if $\phi$ does not satisfy $\Delta_{2}$, then there exists an infinite sequence $\left(f_{i}\right)$ of functions in $L_{\phi}$ of disjoint supports with $I_{\phi}\left(f_{i}\right) \leq \frac{1}{2^{i}}$ and $\left\|f_{i}\right\|=1, i \in \mathbb{N}$. Indeed, if $\phi$ does not satisfy condition $\Delta_{2}$ then by 1.2 there exists a sequence $\left(u_{n}\right) \subset \mathbb{R}_{+}$such that

$$
\phi\left(\left(1+\frac{1}{n}\right) u_{n}\right) \geq 2^{n} \phi\left(u_{n}\right), \quad n \in \mathbb{N} .
$$

Without loss of generality we can assume that $\left(u_{n}\right)$ is increasing. Since $\mu$ is non-atomic and infinite, for every $i \in \mathbb{N}$ there exists an infinite sequence $\left(A_{n}^{i}\right)$ such that

$$
\bigcup_{n=1}^{\infty} A_{n}^{i} \cap \bigcup_{n=1}^{\infty} A_{n}^{j}=\emptyset \quad \text { if } i \neq j
$$

and

$$
\phi\left(u_{n}\right) \mu\left(A_{n}^{i}\right)=\frac{1}{2^{n}} \quad \text { for every } i, n \in \mathbb{N} .
$$


Define

$$
f_{i}=\sum_{n=i+1}^{\infty} u_{n} \chi_{A_{n}^{i}}, \quad n \in \mathbb{N}
$$

Then

$$
I_{\phi}\left(f_{i}\right)=I_{\phi}\left(\sum_{n=i+1}^{\infty} u_{n} \chi_{A_{n}^{i}}\right)=\sum_{n=i+1}^{\infty} \frac{1}{2^{n}}=\frac{1}{2^{i}},
$$

and for any $\lambda>1$ there exists $n_{0}$ such that $\lambda>1+\frac{1}{n}$ for $n \geq n_{0}$. Thus by 1.3 and (1.4) we get

$$
I_{\phi}\left(\lambda f_{i}\right) \geq \sum_{n=n_{0}}^{\infty} \phi\left(\left(1+\frac{1}{n}\right) u_{n}\right) \mu\left(A_{n}^{i}\right) \geq \sum_{n=n_{0}}^{\infty} \frac{2^{n} \phi\left(u_{n}\right)}{2^{n} \phi\left(u_{n}\right)}=\infty .
$$

Consequently

$$
\left\|f_{i}\right\|=1, \quad \text { and } \quad f_{i} \wedge f_{j}=0, \quad \text { for } i \neq j .
$$

Hence and in view of 1.5 ,

$$
I_{\phi}\left(\sum_{i=1}^{\infty} f_{i}\right)=\sum_{i=1}^{\infty} \frac{1}{2^{i}}=1
$$

It follows

$$
\left\|\sum_{n=1}^{\infty} f_{i}\right\|=\left\|f_{i}\right\|=1, \quad \text { for } i \in \mathbb{N} .
$$

Therefore for any $x=\{x(n)\} \in \ell_{\infty}, n \in \mathbb{N}$,

$$
|x(n)|=\left\|x(n) f_{n}\right\| \leq\left\|\sum_{n=1}^{\infty} x(n) f_{n}\right\| \text { and }\left\|\sum_{n=1}^{\infty} x(n) f_{n}\right\| \leq \sup _{n}|x(n)|\left\|\sum_{n=1}^{\infty} f_{n}\right\|=\sup _{n}|x(n)| .
$$

Hence

$$
\left\|\sum_{n=1}^{\infty} x(n) f_{n}\right\|=\sup _{n}|x(n)|=\|x\|_{\infty}
$$

and thus the closure of the linear span of $\left(f_{n}\right)$ in $L_{\phi}$ is lattice isomorphically isometric to $\ell_{\infty}$.

Lemma 1.12. Conditions $\Delta^{q}$ and $\Delta^{* p}, p, q>0$, are preserved under equivalence relation of Orlicz functions.

Proof. We will show this only for $\Delta^{* p}$ when the equivalence relation and the condition are satisfied for all arguments. Let $\phi$ satisfy condition $\Delta^{* p}$ and $\psi$ be equivalent to $\phi$ that is $K_{1}^{-1} \psi\left(K_{2}^{-1} u\right) \leq \phi(u) \leq K_{1} \psi\left(K_{2} u\right)$ for all $u \geq 0$ and some $K_{1}, K_{2} \geq 1$. Then for all $w=K_{2}^{-1} u \geq 0$ and $a \geq K_{2}^{2}$,

$$
\begin{aligned}
K_{1}^{-1} \psi(a w) & =K_{1}^{-2} K_{1} \psi\left(K_{2} a K_{2}^{-2} u\right) \geq K_{1}^{-2} \phi\left(a K_{2}^{-2} u\right) \\
& \geq K_{1}^{-2} K a^{p} K_{2}^{-2 p} \phi(u) \geq K_{1}^{-3} K a^{p} K_{2}^{-2 p} \psi(w) .
\end{aligned}
$$

If $1 \leq a \leq K_{2}^{2}$, then for any $w \geq 0$,

$$
\psi(a w) \geq \psi(w) \geq K_{2}^{-2 p} a^{p} \psi(w) .
$$

Thus we showed that $\psi$ satisfies condition $\Delta^{* p}$. 
The next two results demonstrate possible regularization of $\phi$ satisfying $\Delta^{p}$ or $\Delta^{* p}$. The earliest source of these theorems is in [15]. The thorough studies of these can be found in [9].

Proposition 1.13. Given $q>0$, the following assertions are equivalent.

(a) An Orlicz function (resp. for $q \geq 1$, convex Orlicz function) $\phi$ satisfies condition $\Delta^{q}$.

(b) There exists an Orlicz function (resp. for $q \geq 1$, convex Orlicz function) $\psi$ equivalent to $\phi$ such that for all $u \geq 0, a \geq 1$,

$$
\psi(a u) \leq a^{q} \psi(u) .
$$

(c) There exists an Orlicz function (resp. for $q \geq 1$, convex Orlicz function) $\psi$ equivalent to $\phi$ such that $\psi\left(u^{1 / q}\right)$ is concave.

Proof. (a) $\Rightarrow$ (c) Let do it first for $\phi$ satisfying $\Delta^{q}$-condition for large arguments. Define

$$
r(u)= \begin{cases}\phi(v) / v^{q}, & \text { for } 0 \leq u \leq v \\ \inf _{v \leq t \leq u} \phi(t) / t^{q}, & \text { for } u>v\end{cases}
$$

where $v$ is a constant from condition $\Delta^{q}$ for large arguments. Clearly, $r(u)$ is decreasing and continuous and moreover for every $u \geq v$,

$$
\frac{1}{K} \frac{\phi(u)}{u^{q}} \leq r(u) \leq \frac{\phi(u)}{u^{q}},
$$

where $K$ is a constant in condition $\Delta^{q}$. Set

$$
\psi(u)=\int_{0}^{u} r(t) t^{q-1} d t
$$

Clearly $\psi$ is an Orlicz function.

If $\phi$ is convex and $q \geq 1$, then the function $\psi$ is also convex since $r(t) t^{q-1}$ is increasing. Indeed, it is constant on $(0, v]$, and for $v \leq u_{1}<u_{2}$ we have

$$
r\left(u_{1}\right) u_{1}^{q-1}=u_{1}^{q-1} \inf _{v \leq t \leq u_{1}} \frac{\phi(t)}{t^{q}} \leq u_{2}^{q-1} \inf _{v \leq t \leq u_{1}} \frac{\phi(t)}{t^{q}},
$$

while by 1.6 and in view of that $\phi(u) / u$ is increasing,

Hence

$$
r\left(u_{1}\right) u_{1}^{q-1} \leq \frac{\phi\left(u_{1}\right)}{u_{1}}=u_{2}^{q-1} \inf _{u_{1} \leq t \leq u_{2}} \frac{\phi\left(u_{1}\right)}{t^{q-1} u_{1}} \leq u_{2}^{q-1} \inf _{u_{1} \leq t \leq u_{2}} \frac{\phi(t)}{t^{q}} .
$$

$$
r\left(u_{1}\right) u_{1}^{q-1} \leq u_{2}^{q-1} \min \left\{\inf _{v \leq t \leq u_{1}} \frac{\phi(t)}{t^{q}}, \inf _{u_{1} \leq t \leq u_{2}} \frac{\phi(t)}{t^{q}}\right\}=r\left(u_{2}\right) u_{2}^{q-1} .
$$

In order to check that $\psi$ is equivalent to $\phi$, observe by (1.6) that for $u \geq 2 v$,

$$
\psi(u) \geq r(u) \int_{v}^{u} t^{q-1} d t \geq \frac{1}{q}\left(1-\frac{1}{2^{q}}\right) r(u) u^{q} \geq \frac{1}{q}\left(1-\frac{1}{2^{q}}\right) \frac{1}{K} \phi(u),
$$

which is half of what is needed. For the other inequality, note that in view of 1.6 for $u \geq v$,

$$
\psi(u) \leq \int_{0}^{v} \frac{\phi(v)}{v^{q}} t^{q-1} d t+\int_{v}^{u} \frac{\phi(u)}{u^{q}} t^{q-1} d t \leq \frac{1}{q}(\phi(v)+\phi(u)) \leq \frac{2}{q} \phi(u) .
$$

Thus $\phi$ is equivalent to $\psi$. Next, an easy change of variables yields that $\psi\left(u^{1 / q}\right)=$ $\frac{1}{q} \int_{0}^{u} r\left(t^{1 / q}\right) d t$. Since $r\left(t^{1 / q}\right)$ is decreasing, we conclude that the function $\psi\left(u^{1 / q}\right)$ is concave. 
Since the case "for all arguments" is an easy repetition of the case "for large arguments" by setting $v=0$, what remains to examine is the case when condition $\Delta^{q}$ is satisfied near zero. If we find an Orlicz function $\rho$ equivalent to $\phi$ and satisfying condition $\Delta^{q}$ for all arguments, then the proof can be done by the first step. Define

$$
\rho(u)= \begin{cases}\phi(u), & \text { for } 0 \leq u \leq v \\ c u^{q}+\left(\phi(v)-c v^{q}\right), & \text { for } u>v,\end{cases}
$$

where $v$ is the number in condition $\Delta^{q}$ for small arguments. In case of non-convex $\phi$, let $c>0$ be any constant, and in case of convex $\phi$, set $c=\frac{\phi^{\prime}(v)}{q v^{q-1}}$ where $\phi^{\prime}(v)$ is a left derivative of $\phi$ at $v$. Clearly, $\rho$ is increasing and equivalent to $\phi$ for small arguments, so it is an Orlicz function. If $\phi$ is convex then $\rho$ is also convex.

Now it is enough to show that $\rho(a u) \leq K a^{q} \rho(u)$ for all $a \geq 1, u \geq 0$ and some positive constant $K$. From the assumption of $\Delta^{q}$ for small arguments this inequality is obvious if $a u \leq v$. In the case when $a u>u>v$ and $\phi(v)-c v^{q}>0$,

$$
\rho(a u) \leq a^{q}\left[c u^{q}+\left(\phi(v)-c v^{q}\right)\right]=a^{q} \rho(u) .
$$

When $a u>u>v$ and $b=-\phi(v)+c v^{q}>0$, then

$$
\frac{\rho(a u)}{a^{q} \rho(u)} \leq \frac{c a^{q} u^{q}}{a^{q}\left(c u^{q}-b\right)}=\frac{c u^{q}}{c u^{q}-b}=1+\frac{b}{c u^{q}-b} \leq 1+\frac{b}{c v^{q}-b}=1+\frac{b}{\phi(v)}=k_{0},
$$

where $k_{0}$ does not depend on $u$. Finally for $u \leq v$ and $a u>v$ we get the estimate

$$
\rho(a u) \leq c a^{p} u^{q}+\phi(v) \leq a^{q} u^{q}\left(c+\frac{\phi(v)}{v^{q}}\right)=k_{1} a^{q} u^{q},
$$

with $k_{1}=c+\frac{\phi(v)}{v^{q}}$ is not dependent on $u$. Applying condition $\Delta^{q}$ for $a=\frac{v}{u} \geq 1$ and $u \leq v$ we get

$$
\phi(v)=\phi\left(\frac{v}{u} u\right) \leq K\left(\frac{v}{u}\right)^{q} \phi(u)
$$

which yields $u^{q} \leq \frac{K v^{q}}{\phi(v)} \phi(u)$. Hence

$$
\rho(a u) \leq k_{1} a^{q} u^{q} \leq k_{2} a^{q} \rho(u),
$$

where $k_{2}=\frac{k_{1} K v^{q}}{\phi(v)}>0$ does not depend on $u$. Combining $\sqrt{1.7}, \sqrt{1.8}$ and $(1.9)$ we proved that $\rho \in \Delta^{q}$.

(c) $\Rightarrow$ (b) If $\psi\left(u^{1 / q}\right)$ is concave then the inequality in (b) is instant.

(b) $\Rightarrow$ (a) The inequality in (b) means the $\Delta^{q}$-condition for all arguments of $\psi$. Since $\phi$ is equivalent with $\psi$, by Lemma $1.12 \phi$ also satisfies $\Delta^{q}$-condition.

Proposition 1.14. Given $p>0$, the following conditions are equivalent.

(a) The Orlicz (resp. for $p \geq 1$, convex Orlicz) function $\phi$ satisfies condition $\Delta^{* p}$.

(b) There exists an Orlicz (resp. for $p \geq 1$, convex Orlicz) function $\psi$ equivalent to $\phi$ such that for all $a \geq 1, u \geq 0$

$$
\psi(a u) \geq a^{p} \psi(u) .
$$

(c) There exists an Orlicz (resp. for $p \geq 1$, convex Orlicz) function $\psi$ equivalent to $\phi$ such that $\psi\left(u^{1 / p}\right)$ is convex. 
Proof. Since this result is a dual part of Proposition 1.13, the proof is analogous. We shall show only the essential construction part of implication $(a) \Rightarrow(c)$, in the case where the conditions are satisfied for large arguments. Define

$$
r(u)= \begin{cases}\phi(v) / v^{p}, & \text { for } 0 \leq u \leq v \\ \sup _{v \leq t \leq u} \phi(t) / t^{p}, & \text { for } u>v\end{cases}
$$

where $v$ is a constant in condition $\Delta^{* p}$. The function $r(u)$ is increasing and satisfies the inequality

$$
\frac{\phi(u)}{u^{p}} \leq r(u) \leq \frac{1}{K} \frac{\phi(u)}{u^{p}}
$$

with $K$ from condition $\Delta^{* p}$. By letting

$$
\psi(u)=\int_{0}^{u} r(t) t^{p-1} d t
$$

$\psi$ is an Orlicz function. If $\phi$ is convex and $p \geq 1$ then $\psi$ is also convex since $r(w) w^{p-1}$ is increasing. By changing variables

$$
\psi\left(u^{1 / p}\right)=\frac{1}{p} \int_{0}^{u} r\left(t^{1 / p}\right) d t
$$

and clearly $r\left(t^{1 / p}\right)$ is increasing, so $\psi\left(u^{1 / p}\right)$ is also convex. It remains to check that $\phi$ and $\psi$ are equivalent. By 1.10 for $u \geq 2 v$,

$$
\psi(u) \geq \int_{u / 2}^{u} r(t) t^{p-1} d t \geq r\left(\frac{u}{2}\right) \int_{u / 2}^{u} t^{p-1} d t \geq \frac{\phi(u / 2)}{(u / 2)^{p}}\left(\frac{1}{p}-\frac{1}{2^{p} p}\right) u^{p}=\phi\left(\frac{u}{2}\right) \frac{2^{p}-1}{p} .
$$

For the opposite inequality observe that by condition $\Delta^{* p}$ we have that $\frac{1}{K} \frac{\phi(u)}{u^{p}} \geq \frac{\phi(t)}{t^{p}}$ for $t \leq u$. Letting $u \geq v$,

$$
\begin{aligned}
\psi(u) \leq \int_{0}^{v} \frac{\phi(v)}{v^{p}} t^{p-1} d t+\frac{1}{K} \int_{v}^{u} \frac{\phi(t)}{t^{p}} & t^{p-1} d t \\
& \leq \frac{\phi(v)}{p}+\frac{1}{K^{2}} \int_{v}^{u} \frac{\phi(u)}{u^{p}} t^{p-1} d t \leq \frac{1}{p K^{2}} \phi(u) .
\end{aligned}
$$

REMARK 1.15. In the case of convex Orlicz function and $p \geq 1$, the method used in Propositions 1.131 .14 produces function $\psi$ which is not only convex but also smooth.

There exist several other constructions of $\psi$ (e.g. [1, 9]). If for instance in the proof of Proposition 1.14 we define the function $r(u)$ as

$$
r(u)= \begin{cases}K \phi(v) / v^{p}, & \text { for } 0 \leq u \leq v \\ \sup _{t \geq u} \phi(t) / t^{p}, & \text { for } u>v\end{cases}
$$

then $r(u)$ may not be continuous and thus $\psi$ will not be smooth.

Proposition 1.16. For $1<p<\infty$, a convex Orlicz function $\phi$ satisfies condition $\Delta^{* p}$ if and only if $\phi^{*}$ satisfies condition $\Delta^{p^{\prime}}$, where $\frac{1}{p}+\frac{1}{p^{\prime}}=1$.

Proof. Since in view of Lemma 1.12 both conditions $\Delta^{* p}$ and $\Delta^{p^{\prime}}$ are preserved under equivalence relation, assuming that $\phi$ satisfies $\Delta^{* p}$, by Proposition 1.14 we suppose that 
$\phi(a u) \geq a^{p} \phi(u)$ for every $a \geq 1, u \geq 0$. Hence

$$
\begin{aligned}
\phi^{*}(a u)=\sup _{w>0}\{a u w-\phi(w)\} \geq \sup _{w>0}\left\{a u w-a^{-p} \phi(a w)\right\} & \\
& =\sup _{w>0}\left\{u w-a^{-p} \phi(w)\right\}=a^{-p} \phi^{*}\left(a^{p} u\right),
\end{aligned}
$$

which implies that $\phi^{*}\left(a^{p} u\right) \leq a^{p} \phi^{*}(a u)$ or $\phi^{*}\left(a^{p-1} u\right) \leq a^{p} \phi^{*}(u)$ for every $a \geq 1$ and $u \geq 0$. Letting $b=a^{p-1}, b \geq 1$, we have $b^{p^{\prime}}=a^{p}$ and

$$
\phi^{*}(b u) \leq b^{p^{\prime}} \phi^{*}(u)
$$

for every $b \geq 1$ and $u \geq 0$, and so $\phi^{*}$ satisfies condition $\Delta^{p^{\prime}}$. The converse implication can be shown in a similar fashion.

Proposition 1.17.

(I) Let $\phi$ be an Orlicz function $\phi$. The following conditions are equivalent.

(1) $\phi$ satisfies condition $\Delta_{2}$.

(2) $\phi$ satisfies condition $\Delta^{q}$ for some $0<q<\infty$.

(3) $\beta_{\phi}<\infty$.

(II) Let $\phi$ be a convex Orlicz function. The following conditions are equivalent.

$\left(1^{\prime}\right) \phi^{*}$ satisfies condition $\Delta_{2}$.

$\left(2^{\prime}\right) \phi$ satisfies condition $\Delta^{* p}$ for some $p>1$.

$\left(3^{\prime}\right) \alpha_{\phi}>1$.

Proof.

(I) The equivalence of (2) and (3) follows from Remark 1.10

The implication from (2) to (1) is obvious.

It is enough to show (1) implies (2). We shall prove this only in the case when the relations are satisfied for large arguments. The inequality $\phi(2 u) \leq K \phi(u)$ is valid for all $u \geq v$, where $v \geq 0$ and $K>1$ are some constants. Then for any $a \geq 1$ there exists $m \in \mathbb{N}$ such that $2^{m-1} \leq a<2^{m}$. Setting $q=\ln K / \ln 2$, for every $u \geq v$ and any $a \geq 1$,

$$
\phi(a u) \leq \phi\left(2^{m} u\right) \leq K^{m} \phi(u)=\left(2^{m}\right)^{q} \phi(u) \leq 2^{q} a^{q} \phi(u),
$$

which shows that $\phi$ satisfies condition $\Delta^{q}$.

(II) From part (I), $\phi^{*}$ satisfies $\Delta_{2}$ if and only if $\phi^{*}$ satisfies $\Delta^{q}$ for some $1<q<\infty$. By the assumption that $\phi$ is convex $\phi=\phi^{* *}$. Thus by Proposition 1.16, $\phi^{*} \in \Delta^{q}$ is equivalent to $\phi \in \Delta^{* p}$, where $1 / p+1 / q=1$. Therefore $\left(1^{\prime}\right)$ is equivalent to $\left(2^{\prime}\right)$.

Finally $\left(2^{\prime}\right)$ is equivalent to $\left(3^{\prime}\right)$ by Remark 1.10

\section{Convexity, concavity, lower- and upper-estimates}

TheOREM 2.1. Let $0<q<\infty$ and $\phi$ be an Orlicz function. Then the following properties are equivalent.

(i) The Orlicz space $L_{\phi}$ is q-concave.

(ii) $L_{\phi}$ satisfies a lower q-estimate.

(iii) $\phi$ satisfies condition $\Delta^{q}$. 
Proof. Since for disjointly supported functions $f_{1}, \ldots, f_{n} \in L_{\phi}$,

$$
\left\|\sum_{i=1}^{n} f_{i}\right\|=\left\|\left(\sum_{i=1}^{n}\left|f_{i}\right|^{q}\right)^{1 / q}\right\|
$$

clearly (i) implies (ii).

Assume (ii) and show (iii). Let $L_{\phi}$ satisfy a lower $q$-estimate.

Let us prove it first when the measure $\mu$ is non-atomic and finite. Without loss of generality assume that $\phi(1)=1$ and $\mu(\Omega)=1$. For arbitrary $u>w \geq 1$, set

$$
x=\frac{1}{\phi(u)} \quad \text { and } \quad y=\frac{1}{\phi(w)}
$$

and let $n=\left[\frac{y}{x}\right]$ be the entire part of $\frac{y}{x}$. Clearly $0<x<y \leq 1$ and $n \leq \frac{y}{x} \leq 2 n$. Since $n x \leq y \leq 1$, we are able to choose $n$ disjoint measurable sets $A_{i}$ with $\mu A_{i}=x$ for every $i=1, \ldots, n$. Now, setting

$$
f_{i}=\phi^{-1}(1 / x) \chi_{A_{i}} \quad i=1, \ldots, n,
$$

where $\phi^{-1}$ is an inverse function of $\phi$ on $(0, \infty)$, we obtain that $\left\|f_{i}\right\|=1$ and

$$
\left\|\sum_{i=1}^{n} f_{i}\right\|=\phi^{-1}\left(\frac{1}{x}\right)\left\|\chi_{\bigcup_{i=1}^{n} A_{i}}\right\|=\frac{\phi^{-1}(1 / x)}{\phi^{-1}(1 /(n x))} .
$$

If $D_{q}$ is a lower $q$-estimate constant then in view of $\frac{1}{y} \leq \frac{1}{n x}$,

$$
\begin{aligned}
& \frac{u}{w}=\frac{\phi^{-1}(1 / x)}{\phi^{-1}(1 / y)} \geq \frac{\phi^{-1}(1 / x)}{\phi^{-1}(1 /(n x))}=\left\|\sum_{i=1}^{n} f_{i}\right\| \\
& \quad \geq D_{q}\left(\sum_{i=1}^{n}\left\|f_{i}\right\|^{q}\right)^{1 / q}=D_{q} n^{1 / q} \geq k\left(\frac{y}{x}\right)^{1 / q}=k\left(\frac{\phi(u)}{\phi(w)}\right)^{1 / q},
\end{aligned}
$$

with $k=D_{q} 2^{-1 / q}$. This implies that for every $u>w \geq 1$,

$$
\phi(u) \leq k^{-q}(u / w)^{q} \phi(w),
$$

and thus setting $s=w$ and $a s=u$ where $a \geq 1$ and $s \geq 1$, we obtain

$$
\phi(a s) \leq k^{-q} a^{q} \phi(s),
$$

which means $\Delta^{q}$ condition of $\phi$ for large arguments.

When the measure is non-atomic and infinite, then the proof is similar and in fact simpler.

Let us sketch the proof in the case of discrete measure. Assume also that $\phi(1)=1$. For any $0<w<u \leq 1$, let $x=\frac{1}{\phi(u)}$ and $y=\frac{1}{\phi(w)}$ and $n=\left[\frac{y}{x}\right]$. There exists $m \in \mathbb{N}$ with $m \leq x<m+1$. Choose $n$ disjoint subsets $N_{i}$ of $\mathbb{N}$ with card $N_{i}=m$ and define

$$
x_{i}(j)= \begin{cases}\phi^{-1}(1 / x), & \text { for } j \in N_{i} \\ 0, & \text { for } j \notin N_{i},\end{cases}
$$

for $i=1, \ldots, n$. Then the vectors $x_{i} \in l_{\phi}$ have disjoint supports and $\left\|x_{i}\right\|=\frac{\phi^{-1}(1 / x)}{\phi^{-1}(1 / m)}$.

By the general assumption $\alpha_{\phi}>0$, in view of Remark 1.10 and Proposition 1.14 there exists $p>0$ and equivalent Orlicz function $\psi$ to $\phi$ such that $\psi\left(u^{1 / p}\right)$ is convex. Without 
loss of generality we can assume that $\phi\left(u^{1 / p}\right)$ is convex. It follows that the function $\phi^{-1}\left(u^{p}\right) / u$ is decreasing. Consequently,

$$
\left\|x_{i}\right\|=\frac{\phi^{-1}(1 / x)}{\phi^{-1}(1 / m)} \geq \frac{\phi^{-1}(1 /(m+1))}{\phi^{-1}(1 / m)} \geq\left(\frac{m}{m+1}\right)^{1 / p} .
$$

By

$$
\frac{1}{m n} \geq \frac{1}{y}, \quad n^{1 / q} \geq\left(\frac{1}{2}\right)^{1 / q}\left(\frac{y}{x}\right)^{1 / q},
$$

and by the lower $q$-estimate of $l_{\phi}$,

$$
\begin{aligned}
\frac{u}{w} & =\frac{\phi^{-1}(1 / x)}{\phi^{-1}(1 / y)} \geq \frac{\phi^{-1}(1 / x)}{\phi^{-1}(1 /(n m))}=\left\|\sum_{i=1}^{n} x_{i}\right\| \geq D_{q}\left(\sum_{i=1}^{n}\left(\frac{m}{m+1}\right)^{q / p}\right)^{1 / q} \\
& =D_{q} n^{1 / q}\left(\frac{m}{m+1}\right)^{1 / p} \geq D_{q}\left(\frac{1}{2}\right)^{1 / q+1 / p}\left(\frac{y}{x}\right)^{1 / q}=k\left(\frac{\phi(u)}{\phi(w)}\right)^{1 / q},
\end{aligned}
$$

for every $0<w<u \leq 1$, where $D_{q}$ is the constant of lower $q$-estimate and $k=$ $D_{q}(1 / 2)^{1 / q+1 / p}$ a constant not depending on $x$ and $y$. Similarly as in the first case it implies condition $\Delta^{q}$ for small arguments.

Below an alternative proof is provided in the case of finite non-atomic measure. Observe first that $\phi$ must satisfy condition $\Delta_{2}$. Indeed, in the opposite case in view of Proposition 1.11, $l_{\infty}$ is an order isometric copy in $L_{\phi}$, and so $L_{\phi}$ cannot satisfy any lower $q$-estimate. Note that it is easy to show that $l_{\infty}$ does not have any lower $q$-estimate for any $q<\infty$.

Assume now that $\phi$ does not satisfy condition $\Delta^{q}$ for large arguments. Assume without loss of generality that $\phi(1)=1$. Since $\phi$ does not satisfy $\Delta^{q}$ for large arguments, there exist infinite sequences $\left\{a_{n}\right\}$ and $\left\{u_{n}\right\}$, such that $1 \leq u_{n} \rightarrow \infty$ as $n \rightarrow \infty, a_{n} \geq 1$ and

$$
\phi\left(a_{n}^{1 / q} u_{n}\right) \geq 2^{n} a_{n} \phi\left(u_{n}\right),
$$

for every $n \in \mathbb{N}$. The sequence $\left\{a_{n}\right\}$ is not bounded. Indeed, if for some $M, a_{n} \leq M$ for all $n \in \mathbb{N}$, then by $\Delta_{2}$ condition,

$$
2^{n} a_{n} \phi\left(u_{n}\right) \leq \phi\left(a_{n}^{1 / q} u_{n}\right) \leq \phi\left(M^{1 / q} u_{n}\right) \leq K \phi\left(u_{n}\right)
$$

for all $n \in \mathbb{N}$ and some $K>0$, which is a contradiction. Therefore we assume that $\sum_{n=1}^{\infty} \frac{1}{a_{n}}<\infty$, extracting a subsequence if necessary. Denoting by $\left[a_{n}\right]$ the entire part of $a_{n}$ with $\left[a_{0}\right]=0$, let

$$
N_{n}=\left\{1+\sum_{i=0}^{n-1}\left[a_{i}\right], 2+\sum_{i=0}^{n-1}\left[a_{i}\right], \ldots,\left[a_{n}\right]+\sum_{i=0}^{n-1}\left[a_{i}\right]\right\},
$$

for every $n \in \mathbb{N}$. The sequence $\left\{N_{n}\right\}$ is a disjoint partition of the natural numbers $\mathbb{N}$, and each $N_{n}$ contains exactly $\left[a_{n}\right]$ consecutive natural numbers. There will be no loss of generality if we suppose that $\mu(\Omega)=1$. Observe that by 2.1 ,

$$
\sum_{n=1}^{\infty} \frac{\left[a_{n}\right]}{\phi\left(a_{n}^{1 / q} u_{n}\right)} \leq \sum_{n=1}^{\infty} \frac{1}{2^{n}}=1 \text {. }
$$


Therefore for every $n \in \mathbb{N}$ there exists a finite sequence of sets $\left\{A_{i n}\right\}_{i \in N_{n}}$ such that all $A_{\text {in }}$ are disjoint and

$$
\mu\left(A_{i n}\right)=\frac{1}{\phi\left(a_{n}^{1 / q} u_{n}\right)}
$$

for each $i \in N_{n}, n \in \mathbb{N}$. Define

$$
g_{i n}=u_{n} \chi_{A_{i n}}
$$

for $i \in N_{n}$ and $n \in \mathbb{N}$. For every $n,\left\{g_{i n}\right\}_{i \in N_{n}}$ is a finite sequence of functions with the same distributions and such that

$$
I_{\phi}\left(a_{n}^{1 / q} g_{i n}\right)=\phi\left(a_{n}^{1 / q} u_{n}\right) \mu\left(A_{\text {in }}\right)=1 .
$$

Hence $\left\|g_{i n}\right\|=1 / a_{n}^{1 / q}$ for every $i \in N_{n}, n \in \mathbb{N}$. It then implies that for sufficiently large $n \in \mathbb{N}$,

$$
\sum_{i \in N_{n}}\left\|g_{i n}\right\|^{q}=\sum_{i \in N_{n}} \frac{1}{a_{n}}=\frac{\left[a_{n}\right]}{a_{n}} \geq \frac{1}{2} .
$$

On the other hand since $\phi$ satisfies condition $\Delta_{2}$ for large arguments, for every $\lambda>1$ there exists a constant $K$ such that $\phi(\lambda u) \leq K \phi(u)$ for all $u \geq 1$. Therefore by (2.1),

$$
I_{\phi}\left(\lambda \sum_{i \in N_{n}} g_{i n}\right)=\left[a_{n}\right] \phi\left(\lambda u_{n}\right) \mu\left(A_{i n}\right)=\frac{\left[a_{n}\right] \phi\left(\lambda u_{n}\right)}{\phi\left(a_{n}^{1 / q} u_{n}\right)} \leq \frac{\left[a_{n}\right] \phi\left(\lambda u_{n}\right)}{2^{n} a_{n} \phi\left(u_{n}\right)} \leq \frac{K}{2^{n}},
$$

where $K$ depends only on $\lambda$. Hence for all $\lambda>0, I_{\phi}\left(\lambda \sum_{i \in N_{n}} g_{i n}\right) \rightarrow 0$, which means that $\left\|\sum_{i \in N_{n}} g_{i n}\right\| \rightarrow 0$ as $n \rightarrow \infty$. Thus in view of 2.2 , $L_{\phi}$ cannot satisfy any lower $q$-estimate.

We shall show now the implication from (iii) to (i). Let $\phi$ satisfy condition $\Delta^{q}$. In view of Proposition 1.13 there exists an Orlicz function $\psi$ equivalent to $\phi$ such that $\psi\left(u^{1 / q}\right)$ is concave. Since the norms determined by both functions $\psi$ and $\phi$ are equivalent, we assume not losing generality that $\phi\left(u^{1 / q}\right)$ is concave. By direct calculations we get

$$
\|f\|_{\phi\left(u^{1 / q}\right)}=\left\||f|^{1 / q}\right\|_{\phi}^{q} .
$$

Notice also that the Luxemburg functional defined by means of a concave function (e.g. $\left.\|\cdot\|_{\phi\left(u^{1 / q}\right)}\right)$ satisfies the reverse triangle inequality. Thus

$$
\left\|\left(\sum_{i=1}^{n}\left|f_{i}\right|^{q}\right)^{1 / q}\right\|_{\phi}=\left\|\sum_{i=1}^{n}\left|f_{i}\right|^{q}\right\|_{\phi\left(u^{1 / q}\right)}^{1 / q} \geq\left(\sum_{i=1}^{n}\left\|\left|f_{i}\right|^{q}\right\|_{\phi\left(u^{1 / q}\right)}\right)^{1 / q}=\left(\sum_{i=1}^{n}\left\|f_{i}\right\|_{\phi}^{q}\right)^{1 / q}
$$

which shows that $L_{\phi}$ is $q$-concave.

THEOREM 2.2. Let $0<p<\infty$ and $\phi$ be an Orlicz function. Then the following conditions are equivalent.

(i) The Orlicz space $L_{\phi}$ is p-convex.

(ii) $L_{\phi}$ satisfies an upper $p$-estimate.

(iii) $\phi$ satisfies condition $\Delta^{* p}$.

Proof. It is clear that (i) yields (ii).

(ii) $\Rightarrow$ (iii) Let now $L_{\phi}$ satisfy an upper $p$-estimate. We will show that $\phi \in \Delta^{* p}$. We are giving the proof only in the case of finite and non-atomic measure. Assume without 
loss of generality that $\phi(1)=1$ and $\mu(\Omega)=1$. For the sake of convenience we will repeat several steps from the proof of the implication (ii) to (iii) of Theorem 2.1

For arbitrary $u>w \geq 1$, set

$$
x=\frac{1}{\phi(u)} \quad \text { and } \quad y=\frac{1}{\phi(w)}
$$

and let $n=\left[\frac{y}{x}\right]$ be the entire part of $\frac{y}{x}$. Clearly $0<x<y \leq 1$ and $n \leq \frac{y}{x} \leq 2 n$. Since $n x \leq y \leq 1$, we are able to choose $n$ disjoint measurable sets $A_{i}$ with $\mu A_{i}=x$ for every $i=1, \ldots, n$. Now, setting

$$
f_{i}=\phi^{-1}(1 / x) \chi_{A_{i}} \quad i=1, \ldots, n,
$$

where $\phi^{-1}$ is the inverse function on $(0, \infty)$ of $\phi$, we obtain $\left\|f_{i}\right\|=1, f_{i} \wedge f_{j}=0$ for $i \neq j$, and

$$
\left\|\sum_{i=1}^{n} f_{i}\right\|=\phi^{-1}(1 / x)\left\|\chi_{\bigcup_{i=1}^{n} A_{i}}\right\|=\frac{\phi^{-1}(1 / x)}{\phi^{-1}(1 /(n x))} .
$$

The general assumption $\alpha_{\phi}>0$ implies that $\phi^{-1}$ satisfies condition $\Delta_{2}$. Indeed there exist constants $1>C>0, r>0$ such that $\phi(a t) \geq C a^{r} \phi(t)$ for all $a \geq 1$ and $t \geq 0$. Hence for all $s=\phi(t) \geq 0$ we have $a \phi^{-1}(s) \geq \phi^{-1}\left(C a^{r} s\right)$. Setting $K=a=(2 / C)^{r}$, we get

$$
\phi^{-1}(2 s) \leq(2 / C)^{r} \phi^{-1}(s)=K \phi^{-1}(s) .
$$

If $C_{p}$ is an upper $p$-estimate constant then in view of $1 / y \geq 1 /(2 n x)$ and the above inequality,

$$
\begin{aligned}
\frac{u}{w} & =\frac{\phi^{-1}(1 / x)}{\phi^{-1}(1 / y)} \leq \frac{\phi^{-1}(1 / x)}{\phi^{-1}(1 /(2 n x))} \leq K \frac{\phi^{-1}(1 / x)}{\phi^{-1}(1 /(n x))} \\
& =K\left\|\sum_{i=1}^{n} f_{i}\right\| \leq C_{p}\left(\sum_{i=1}^{n}\left\|f_{i}\right\|^{p}\right)^{1 / p}=K C_{p} n^{1 / p} \leq K C_{p}\left(\frac{y}{x}\right)^{1 / p}=K C_{p}\left(\frac{\phi(u)}{\phi(w)}\right)^{1 / p} .
\end{aligned}
$$

This implies that for every $u>w \geq 1$,

$$
\phi(u) \geq\left(K C_{p}\right)^{-p}(u / w)^{p} \phi(w),
$$

and thus setting $s=w$ and $a s=u$ where $a \geq 1$ and $s \geq 1$,

$$
\phi(a s) \geq\left(K C_{p}\right)^{-p} a^{p} \phi(s),
$$

which means $\Delta^{* p}$ condition of $\phi$ for large arguments.

Proof of (iii) $\Rightarrow$ (i) if $\phi$ is convex.

In this case $L_{\phi}$ is a Banach space and we can use general duality between convexity and concavity. In view of Proposition 1.16 $\phi^{*}$ satisfies condition $\Delta^{p^{\prime}}$. Thus by Theorem 2.1. $L_{\phi^{*}}$ must be $p^{\prime}$-concave. Moreover $\phi^{*} \in \Delta^{p^{\prime}}$ implies that $\phi^{*} \in \Delta_{2}$ in view of Proposition 1.17. Therefore $L_{\phi^{*}}=E_{\phi^{*}}$, and so $\left(L_{\phi^{*}}\right)^{*} \simeq L_{\phi}$. Consequently, in view of Theorem 1.4 $L_{\phi}$ is $p$-convex.

Proof of (iii) $\Rightarrow$ (i) for arbitrary Orlicz function $\phi$.

We can give a proof analogous to the last part of the proof in Theorem 2.1 replacing concavity by convexity. However we can treat this more generally. Recall, given $0<$ $p<\infty$ and a quasi-Banach lattice $\left(X,\|\cdot\|_{X}\right)$, the $p$-convexification of $X$ is the space 
$X^{(p)}=\left\{x \in X:|x|^{p} \in X\right\}$ equipped with the quasi-norm $\|x\|_{X^{(p)}}=\left\||x|^{p}\right\|_{X}^{1 / p}$. It is straightforward to show that $X^{(p)}$ is $p$-convex [13, page 53].

Now if $\phi$ satisfies condition $\Delta^{* p}$, then in view of Proposition 1.14 $\phi$ is equivalent to an Orlicz function $\psi$ such that $\psi\left(u^{1 / p}\right)$ is convex. Thus $L_{\psi\left(u^{1 / p}\right)}$ is a Banach space. Moreover, $L_{\psi}=\left(L_{\psi\left(u^{1 / p}\right)}\right)^{(p)}$ is a $p$-convexification of $L_{\psi\left(u^{1 / p}\right)}$. Hence $L_{\psi}$ and so $L_{\phi}$ is p-convex.

\section{Type and cotype}

TheOREM 3.1. Let $q \geq 2$ and $\phi$ be an Orlicz function. Then the Orlicz space $L_{\phi}$ has cotype $q$ if and only if $\phi$ satisfies condition $\Delta^{q}$.

Proof. Under the additional assumption of convexity on $\phi$, the space $L_{\phi}$ is a Banach lattice. Therefore we can use general facts from Banach spaces and lattices. The summary of the relations among several local geometric properties is given in two diagrams in the monograph [13, pp. 100-101]. By them, for $q>2$, lower $q$-estimate is equivalent to cotype $q$. Moreover, 2-concavity and cotype 2 are also equivalent. Thus in view of Theorem 2.1 the proof is done.

Assume now that $\phi$ is an arbitrary Orlicz function, not necessarily convex, and that $L_{\phi}$ has cotype $q<\infty$. We will show that $\phi$ satisfies condition $\Delta^{q}$.

Observe first that $\phi$ must satisfy condition $\Delta_{2}$. Indeed, in the opposite case in view of Proposition 1.11 $L_{\phi}$ contains an isomorphic copy of $l_{\infty}$. The space $l_{\infty}$ does not have any finite cotype. In fact let $e_{i}=(0, \ldots, 0,1,0, \ldots), i \in \mathbb{N}$, with 1 on the $i$-th place, be unit vectors in $l_{\infty}$. Then

$$
\int_{0}^{1}\left\|\sum_{i=1}^{n} r_{i}(t) e_{i}\right\|_{\infty} d t=\left\|\sum_{i=1}^{n} e_{i}\right\|_{\infty}=1 \quad \text { and } \quad \sum_{i=1}^{n}\left\|e_{i}\right\|_{\infty}^{q}=n,
$$

for any $n \in \mathbb{N}$ and $q>0$, which implies that $l_{\infty}$ does not have a finite cotype. It follows that $L_{\phi}$ does not have it either, which contradicts the assumption.

We provide a further proof only in the case of finite non-atomic measure. Assume that $\phi$ does not satisfy condition $\Delta^{q}$ for large arguments. We employ now the functions $\left\{g_{i n}\right\}_{i \in N_{n}}$ constructed in the proof of Theorem 2.1. They satisfy inequalities 2.2 and 2.3 . Hence for all $\lambda>0, I_{\phi}\left(\lambda \sum_{i \in N_{n}} g_{i n}\right) \rightarrow 0$, equivalently $\left\|\sum_{i \in N_{n}} g_{i n}\right\| \rightarrow 0$ as $n \rightarrow \infty$. By this, 2.2 and the assumption that $L_{\phi}$ has cotype $q$,

$$
\frac{1}{2} \leq \sum_{i \in N_{n}}\left\|g_{i n}\right\|^{q} \leq K^{q}\left(\int_{0}^{1}\left\|\sum_{i \in N_{n}} r_{i}(t) g_{i n}\right\| d t\right)^{q}=K^{q}\left\|\sum_{i \in N_{n}} g_{i n}\right\|^{q} \rightarrow 0,
$$

as $n \rightarrow \infty$, which concludes that $L_{\phi}$ has no cotype $q$, and thus $\phi \in \Delta^{q}$.

Let now $\phi$ satisfy condition $\Delta^{q}$. Then by Theorem 2.1 $L_{\phi}$ is $q$-concave.

By the general assumption $\alpha_{\phi}>0$ and Remark 1.10 $\phi \in \Delta^{* r}$ for some $r>0$. This yields that $L_{\phi}$ is $r$-convex by Theorem 2.2 Therefore in view of the generalization of Khintchine's inequality given by Theorem 1.6 and $2 \leq q<\infty$,

$$
\int_{0}^{1}\left\|\sum_{i=1}^{n} r(t) f_{i}\right\| d t \geq C\left\|\left(\sum_{i=1}^{n}\left|f_{i}\right|^{2}\right)^{1 / 2}\right\| \geq C\left\|\left(\sum_{i=1}^{n}\left|f_{i}\right|^{q}\right)^{1 / q}\right\| \geq C D_{q}\left(\sum_{i=1}^{n}\left\|f_{i}\right\|^{q}\right)^{1 / q},
$$

where $D_{q}$ is a $q$-concavity constant of $L_{\phi}$. Hence $L_{\phi}$ has cotype $q$. 
Proposition 3.2. Let $0<p \leq 2$. If $L_{\phi}$ has type $p$ then $\phi$ satisfies condition $\Delta^{* p}$.

Proof. We provide a proof only in the case of finite non-atomic measure. Assume without loss of generality that $\phi(1)=1$ and $\mu(\Omega)=1$.

Assume that $\phi$ does not satisfy condition $\Delta^{* p}$ for large arguments. There exist infinite sequences $\left\{a_{n}\right\}$ and $\left\{u_{n}\right\}$, such that $1 \leq u_{n} \rightarrow \infty$ as $n \rightarrow \infty, a_{n} \geq 1$ and

$$
\phi\left(a_{n}^{1 / p} u_{n}\right) \leq a^{-n} a_{n} \phi\left(u_{n}\right),
$$

for every $n \in \mathbb{N}$ and some $a \geq 2$. Observe that the sequence $\left\{a_{n}\right\}$ is not bounded. If for a contrary, for some $K>0, a_{n} \leq K$ for all $n \in \mathbb{N}$, then

$$
\phi\left(u_{n}\right) \leq \phi\left(a_{n}^{1 / p} u_{n}\right) \leq a^{-n} a_{n} \phi\left(u_{n}\right) \quad \text { so } \quad 1 \leq \frac{a_{n}}{a^{n}} \leq \frac{K}{a^{n}} \rightarrow 0 \quad \text { as } \quad n \rightarrow \infty,
$$

which is a contradiction. Recall, given $a \in \mathbb{R}$, the ceiling function $\lceil a\rceil$ is the smallest integer bigger than or equal to $a$. Let $\left\lceil a_{0}\right\rceil=0$ and

$$
N_{n}=\left\{1+\sum_{i=0}^{n-1}\left\lceil a_{i}\right\rceil, 2+\sum_{i=0}^{n-1}\left\lceil a_{i}\right\rceil, \ldots,\left\lceil a_{n}\right\rceil+\sum_{i=0}^{n-1}\left\lceil a_{i}\right\rceil\right\},
$$

for every $n \in \mathbb{N}$. The sequence $\left\{N_{n}\right\}$ is a disjoint partition of natural numbers $\mathbb{N}$, and each $N_{n}$ contains exactly $\left\lceil a_{n}\right\rceil$ consecutive natural numbers. By the assumption $\alpha_{\phi}>0$, in view of Remark 1.10, $\phi \in \Delta^{* s}$ for some $p>s>0$. Further by Proposition 1.14 the function $\phi\left(u^{1 / s}\right)$ is equivalent to a convex function. Assume thus not losing generality that $\phi\left(u^{1 / s}\right)$ is convex. Thus by 3.2 ,

$$
\left\lceil a_{n}\right\rceil \phi\left(u_{n}\right) \geq 2^{n} \phi\left(a_{n}^{1 / p} u_{n}\right)=2^{n} \phi\left(\left(a_{n}^{s / p} u_{n}^{s}\right)^{1 / s}\right) \geq \phi\left(2^{n / s} a_{n}^{1 / p} u_{n}\right) .
$$

Clearly

$$
\frac{\left\lceil a_{n}\right\rceil}{\phi\left(2^{n / s} a_{n}^{1 / p} u_{n}\right)} \leq \frac{a_{n}+1}{\phi\left(2^{n / s} a_{n}^{1 / p}\right)} .
$$

Consider now the convex function $\psi(u)=\phi\left(u^{1 / s}\right)$. It follows that $\psi\left(2^{x} x^{s / p}\right) \geq 2^{x} x^{s} \psi(1)$ for $x \geq 1$. Therefore

$$
\lim _{x \rightarrow \infty} \frac{x+1}{\phi\left(2^{x / s} x^{1 / p}\right)}=\lim _{x \rightarrow \infty} \frac{x+1}{\psi\left(2^{x} x^{s / p}\right)} \leq \lim _{x \rightarrow \infty} \frac{2 x}{2^{x} x^{s / p} \psi(1)}=\lim _{x \rightarrow \infty} \frac{2 x^{1-\frac{s}{p}}}{2^{x} \psi(1)}=0 .
$$

Hence choosing a subsequence if necessary we can assume that

$$
\sum_{n=1}^{\infty} \frac{\left\lceil a_{n}\right\rceil}{\phi\left(2^{n / s} a_{n}^{1 / p} u_{n}\right)}<\mu(\Omega)=1 .
$$

Therefore for every $n \in \mathbb{N}$ there exists a finite sequence of disjoint sets $\left\{A_{i n}\right\}_{i \in N_{n}} \subset \Omega$ such that

$$
\mu\left(A_{\text {in }}\right)=\frac{1}{\phi\left(2^{n / s} a_{n}^{1 / p} u_{n}\right)}
$$

for each $i \in N_{n}, n \in \mathbb{N}$. Defining for $i \in N_{n}$ and $n \in \mathbb{N}$,

$$
g_{\text {in }}=u_{n} \chi_{A_{i n}},
$$

it follows that

$$
I_{\phi}\left(2^{n / s} a_{n}^{1 / p} g_{i n}\right)=\phi\left(2^{n / s} a_{n}^{1 / p} u_{n}\right) \mu\left(A_{\text {in }}\right)=1 .
$$


Consequently,

$$
\left\|g_{i n}\right\|^{p}=\frac{1}{2^{n p / s} a_{n}} \quad \text { and } \quad \sum_{i \in N_{n}}\left\|g_{i n}\right\|^{p}=\frac{\left\lceil a_{n}\right\rceil}{2^{n p / s} a_{n}} \leq \frac{2}{2^{n p / s}} \rightarrow 0,
$$

as $n \rightarrow \infty$. On the other hand by 3.3 , for every $n \in \mathbb{N}$,

$$
I_{\phi}\left(\sum_{i \in N_{n}} g_{i n}\right)=\left\lceil a_{n}\right\rceil \phi\left(u_{n}\right) \mu\left(A_{i n}\right)=\frac{\left\lceil a_{n}\right\rceil \phi\left(u_{n}\right)}{\phi\left(2^{n / s} a_{n}^{1 / p} u_{n}\right)} \geq 1 .
$$

Now since $g_{i n}$ are disjointly supported for $i \in N_{n}$, we get

$$
K\left(\sum_{i \in N_{n}}\left\|g_{i n}\right\|^{p}\right)^{1 / p} \geq \int_{0}^{1}\left\|\sum_{i \in N_{n}} r_{i}(t) g_{i n}\right\| d t=\left\|\sum_{i \in N_{n}} g_{i n}\right\| \geq 1 .
$$

Finally combining (3.4) and (3.5), $L_{\phi}$ cannot have type $p$, which finishes the proof.

Proposition 3.3. Let $1<p \leq 2$. If $L_{\phi}$ has type $p$ then $\phi$ satisfies condition $\Delta_{2}$.

Proof. If $\phi$ does not satisfy $\Delta_{2}$ then by Proposition 1.11 $L_{\phi}$ contains an isomorphic copy of $l_{\infty}$, which by reasoning as in 3.1 , does not have any finite cotype. Thus $L_{\phi}$ does not possess any finite cotype either. Now observe that $L_{\phi}$ can be treated as a Banach space. Indeed if $L_{\phi}$ has type $p>1$ then by Theorem 1.3 , the space $L_{\phi}$ is normable. Consequently in view of Theorem 1.5 it cannot have type bigger than 1 .

THEOREM 3.4. Given $1<p \leq 2$ and an Orlicz function $\phi$, the Orlicz space $L_{\phi}$ has type $p$ if and only if $\phi$ satisfies both conditions $\Delta_{2}$ and $\Delta^{* p}$.

Proof.

Proof in the case $1<p \leq 2$ and a convex $\phi$.

Here we use general relations in Banach spaces between type and convexity, and duality between type and cotype.

Let $\phi$ satisfy $\Delta_{2}$ and $\Delta^{* p}$. It follows that $L_{\phi}$ is $p$-convex by Theorem 2.2. Moreover, by Proposition 1.17, $\phi \in \Delta^{q}$ for some $1<q<\infty$, and hence $L_{\phi}$ is $q$-concave in view of Theorem 2.1. If we apply now the diagram on page 101 in [13], $L_{\phi}$ has type $p$.

If $L_{\phi}$ has type $p>1$, then due to Theorem 1.5 the space is $q$-concave for some $q<\infty$. Consequently, it cannot contain an order isomorphic copy of $l_{\infty}$ and so by Proposition 1.11 $\phi$ must satisfy condition $\Delta_{2}$. It follows that $\left(L_{\phi}\right)^{*} \simeq L_{\phi^{*}}$. This in turn implies that $L_{\phi^{*}}$ has cotype $p^{\prime}$ from Theorem 1.4 Now by Theorem 3.1. $\phi^{*} \in \Delta^{p^{\prime}}$ and then in view of Proposition 1.16, $\phi \in \Delta^{* p}$.

Proof in the case $1<p \leq 2$ and an arbitrary Orlicz function $\phi$.

The necessity follows from Propositions 3.2 and 3.3

Sufficiency. Let now $\phi \in \Delta_{2}$ and $\phi \in \Delta^{* p}$.

By Theorem 2.2, the condition $\Delta^{* p}$ implies that the space $L_{\phi}$ is $p$-convex. In view of Proposition 1.17 the assumption $\Delta_{2}$ for $\phi$ implies that $\phi \in \Delta^{q}$ for some $q<\infty$, and so $L_{\phi}$ is $q$-concave by Theorem 2.1. Then applying Theorem 1.6 for $L_{\phi}$ and $0<p \leq 2$, for any $f_{1}, \ldots, f_{n} \in L_{\phi}$ we get

$$
\int_{0}^{1}\left\|\sum_{i=1}^{n} r(t) f_{i}\right\| d t \leq C\left\|\left(\sum_{i=1}^{n}\left|f_{i}\right|^{2}\right)^{1 / 2}\right\| \leq C\left\|\left(\sum_{i=1}^{n}\left|f_{i}\right|^{p}\right)^{1 / p}\right\| \leq C\left(\sum_{i=1}^{n}\left\|f_{i}\right\|^{p}\right)^{1 / p} .
$$

Hence $L_{\phi}$ has type $p$. 
REMARK 3.5. Observe that the proof of sufficiency in Theorem 3.4 works for any $0<p \leq 2$.

REMARK 3.6. Kalton [4, Theorem 4.2] showed that for $0<p<1$, any quasi-Banach space is $p$-normable if and only if it has type $p$. For $p=1$ this is no longer true. Clearly any normable space has type 1 , however there exist quasi-Banach spaces that possess type 1 but they are not normable [4. There are also spaces that are neither normable nor of type 1 , as weak- $L^{1}$ spaces considered in [5, 8]. In this respect Orlicz spaces behave more regularly. As we see in the next corollaries, normability is equivalent to type 1 in $L_{\phi}$. Corollary 3.7. Let $\phi$ be an Orlicz function and $0<p \leq 1$. Then (i), (ii) and (iii) are equivalent.

(i) $\phi$ satisfies condition $\Delta^{* p}$.

(ii) $L_{\phi}$ is p-normable.

(iii) $L_{\phi}$ has type $p$.

Proof. (i) $\Rightarrow$ (ii) By Theorem 2.2, $L_{\phi}$ is $p$-convex. Then by the inequality $|a+b|^{p} \leq$ $|a|^{p}+|b|^{p}, a, b \in \mathbb{R}$, we get

$$
\left\|\sum_{i=1}^{n} f_{i}\right\| \leq\left\|\left(\sum_{i=1}^{n}\left|f_{i}\right|^{p}\right)^{1 / p}\right\| \leq C_{p}\left(\sum_{i=1}^{n}\left\|f_{i}\right\|^{p}\right)^{1 / p}
$$

for any $f_{1}, \ldots, f_{n} \in L_{\phi}, n \in \mathbb{N}$. Thus $\|\cdot\|$ is $p$-normable.

(ii) $\Rightarrow$ (iii) Let $f_{1}, \ldots, f_{n} \in L_{\phi}$. By $p$-normability, there exists $C>0$ such that

$$
\int_{0}^{1}\left\|\sum_{i=1}^{n} r_{i}(t) f_{i}\right\| d t \leq\left\|\sum_{i=1}^{n}\left|f_{i}\right|\right\| \leq C\left(\sum_{i=1}^{n}\left\|f_{i}\right\|^{p}\right)^{1 / p} .
$$

(iii) $\Rightarrow$ (i) See Proposition 3.2 .

In view of Proposition 1.14 the immediate consequence of Corollary 3.7 for $p=1$ is the following result.

Corollary 3.8. Let $\phi$ be an Orlicz function. Then (i), (ii) and (iii) are equivalent.

(i) $\phi$ is equivalent to a convex function.

(ii) $L_{\phi}$ is normable.

(iii) $L_{\phi}$ has type 1 .

4. Concluding results. Summarizing the results from three previous sections, Propositions 1.13, 1.14, Theorems 2.1, 2.2, 3.1, 3.4 and Corollary 3.7, we obtain the next two theorems.

TheOREM 4.1. Let $\alpha_{\phi}>0$ and $0<q<\infty$. Consider the following properties.

(i) $\phi$ satisfies condition $\Delta^{q}$.

(ii) There exists an Orlicz function $\psi$ equivalent to $\phi$ such that $\psi\left(u^{1 / q}\right)$ is concave.

(iii) $L_{\phi}$ is q-convex.

(iv) $L_{\phi}$ satisfies a lower q-estimate.

(v) $L_{\phi}$ has cotype $q$.

The conditions (i)-(iv) are equivalent. If $q \geq 2$, then all conditions (i)-(v) are equivalent. 
Theorem 4.2. Let $\alpha_{\phi}>0$ and $0<p<\infty$. Consider the conditions below.

(i) $\phi$ satisfies condition $\Delta^{* p}$.

(ii) There exists an Orlicz function $\psi$ equivalent to $\phi$ such that $\psi\left(u^{1 / p}\right)$ is convex.

(iii) $L_{\phi}$ satisfies an upper $p$-estimate.

(iv) $L_{\phi}$ is p-convex.

(v) $L_{\phi}$ is p-normable.

(vi) $\phi$ satisfies conditions $\Delta_{2}$ and $\Delta^{* p}$.

(vii) $L_{\phi}$ has type $p$.

If $0<p<\infty$ then (i)-(iv) are equivalent.

If $1<p \leq 2$ then (vi)-(vii) are equivalent.

If $0<p \leq 1$, then (i)-(v) and (vii) are equivalent.

For Banach spaces the notions of type and cotype are related to geometric properties of subspaces uniformly isomorphic to finite dimensional spaces $l_{1}^{n}$ and $l_{\infty}^{n}, n \in \mathbb{N}$, respectively. Recall that $l_{1}^{n}$ and $l_{\infty}^{n}$ are $n$-dimensional spaces equipped with $l_{1}$ - and $l_{\infty}$-norm respectively. A Banach space has always type $1 \leq p \leq 2$. If it has only type $p=1$, then we say that it has a trivial type.

COROLlary 4.3. Let $\phi$ be a convex function. The following conditions are equivalent.

(1) $L_{\phi}$ has nontrivial type.

(2) $L_{\phi}$ does not contain $l_{1}^{n \prime} s$ uniformly.

(3) $L_{\phi}$ is B-convex.

(4) $1<\alpha_{\phi} \leq \beta_{\phi}<\infty$, that is $\phi$ and $\phi^{*}$ satisfy condition $\Delta_{2}$.

(5) $L_{\phi}$ is reflexive.

Proof. By the assumption of convexity of $\phi, L_{\phi}$ is a Banach space. The equivalence of (1) and (2) is true for any Banach space by Pisier's Theorem [2, Theorem 13.3], as well as the equivalence of (2) and (3) by [2, Theorem 13.6]. By Theorem 3.4, $L_{\phi}$ has type $1<p \leq 2$ if and only if $\phi \in \Delta^{* p}$ and $\phi \in \Delta_{2}$. By Proposition 1.17 the latter condition is equivalent to $\beta_{\phi}<\infty$, while the previous one is equivalent to $\alpha_{\phi}>1$. Moreover $L_{\phi}$ is reflexive if and only if $\phi$ and $\phi^{*}$ satisfy condition $\Delta_{2}$ [1, 11, 14], so (4) is equivalent to $(5)$.

Corollary 4.4. Let $\phi$ be a convex function. All conditions below are equivalent.

(1) $L_{\phi}$ has a finite cotype.

(2) $L_{\phi}$ does not contain $l_{\infty}^{n}$ 's uniformly.

(3) $\beta_{\phi}<\infty$, that is $\phi$ satisfies condition $\Delta_{2}$.

Proof. For any Banach space, conditions (1) and (2) are equivalent [2, Theorem 14.1]. Moreover by Theorem 3.1, $L_{\phi}$ has cotype $2 \leq q<\infty$ if and only if $\phi \in \Delta^{q}$. In view of Proposition 1.17, the existence of such a $q$, in turn, is equivalent to $\beta_{\phi}<\infty$.

Corollary 4.5. Let $\phi$ be an Orlicz function with $\alpha_{\phi}>0$.

I. The Orlicz space $L_{\phi}$ has cotype $\max \left(\beta_{\phi}+\varepsilon, 2\right)$ for every $\varepsilon>0$. If $L_{\phi}$ has a finite cotype $q$ then $q \geq \max \left(\beta_{\phi}, 2\right)$.

II. The Orlicz space $L_{\phi}$ has type $\min \left(\alpha_{\phi}-\varepsilon, 2\right)$, for every $\varepsilon>0$ with $\alpha_{\phi}-\varepsilon>0$, whenever $\beta_{\phi}<\infty$. If $L_{\phi}$ has a nontrivial type $p>0$, then $\beta_{\phi}<\infty$ and $p \leq \min \left(\alpha_{\phi}, 2\right)$. 
Proof. Part I follows from Remark 1.10 and Theorem 3.1. Part II is a result of Remark 1.10 and Theorem 3.4

EXAmples 4.6. (1) For any $p \in(0,2]$ and $q \in[2, \infty)$, there exists an Orlicz space of type $p$ and cotype $q$.

(a) Let $L_{\phi}$ be induced by $\phi(u)=\max \left(u^{p}, u^{q}\right), 0<p \leq 2 \leq q<\infty$. Then $\phi$ is an Orlicz function not necessarily convex with $\alpha_{\phi}=p$ and $\beta_{\phi}=q$. If $1 \leq p \leq 2$ then $\phi$ is convex and $L_{\phi}$ is a Banach space.

Moreover $L_{\phi}=L_{p} \cap L_{q}$ where $\|\cdot\|_{\phi}$ is a quasi-norm equivalent to $\max \left\{\|\cdot\|_{p},\|\cdot\|_{q}\right\}$. Here $\|f\|_{r}=\left(\int|f|^{r}\right)^{1 / r}, r>0$. It is easy to check that $\phi \in \Delta_{2}$ and $\phi \in \Delta^{* p}$, and $\phi \in \Delta^{q}$. So by Theorem 3.4 it has type $p=\alpha_{\phi}$, and by Theorem 3.1 it has cotype $q=\beta_{\phi}$.

(b) Let $L_{\phi}=L_{p}+L_{q}$, where $\phi(u)=\min \left(u^{p}, u^{q}\right), 0<p \leq 2 \leq q<\infty$. Then $\alpha_{\phi}=p$ and $\beta_{\phi}=q$, and $\|f\|_{\phi}$ is equivalent to $\inf \left\{\|g\|_{p}+\|h\|_{q}: f=g+h, g \in L_{p}, h \in L_{q}\right\}$. Again as above $\phi \in \Delta_{2}$ and $\phi \in \Delta^{* p}$, and $\phi \in \Delta^{q}$. Thus $L_{\phi}$ has type $p=\alpha_{\phi}$ and cotype $q=\beta_{\phi}$.

The function $\phi(u)=\min \left(u^{p}, u^{q}\right)$ is never convex. However if $1 \leq p \leq 2$ then it is equivalent to a convex Orlicz function. In fact $\phi$ is equivalent to

$$
\Phi(u)=\int_{0}^{u} \min \left(t^{p-1}, t^{q-1}\right) d t=\int_{0}^{u} \frac{\phi(t)}{t} d t .
$$

Obviously $\frac{\phi(u)}{u}$ is increasing for $u>0$, so $\Phi$ is convex. Moreover, for every $u>0$,

$$
\phi\left(\frac{u}{2}\right) \leq \Phi(u) \leq \phi(u) .
$$

In this case the quasinorm $\|\cdot\|_{\phi}$ is equivalent to the Luxemburg norm $\|\cdot\|_{\Phi}$, that is $L_{\phi}$ is normable.

If $F: \mathbb{R} \rightarrow \mathbb{R}$ is any function then $F^{*}(v)=\sup _{u \in \mathbb{R}}\{u v-F(u)\}, v \in \mathbb{R}$, is said to be the Legendre transform $F$. It is well known that $F=F^{* *}$ if and only if $F$ is convex [3], Theorem 1, p. 175]. Since $F^{* *} \leq F$ for any $F, F^{* *}$ is a convex minorant of $F$.

In our case $\phi^{* *} \leq \phi$ is a convex minorant of $\phi$. Moreover $\Phi^{* *}=\Phi$, and thus

$$
\phi^{* *}\left(\frac{u}{2}\right) \leq \Phi(u) \leq \phi^{* *}(u) .
$$

Therefore $\phi$ is equivalent to its convex minorant.

(2) An Orlicz space need not have its upper index as a cotype. Let $q \geq 2$ and define

$$
\phi(u)= \begin{cases}0, & \text { for } u=0 \\ \frac{u^{q}}{|\ln u|}, & \text { for } 0<u \leq \frac{1}{e} \\ \left(\frac{1}{q}+1\right) u^{q}-\frac{1}{q} e^{-q}, & \text { for } u>\frac{1}{e} .\end{cases}
$$

Then $\beta_{\phi}=q$, but $\sup _{\lambda \geq 1, u>0} \frac{\phi(\lambda u)}{\lambda^{q} \phi(u)} \geq \sup _{\lambda \geq 1} \frac{|\ln (1 / e)|}{|\ln (\lambda / e)|}=\infty$, which implies that $\phi$ does not satisfy condition $\Delta^{q}$. Thus $L_{\phi}$ has cotype $q+\varepsilon$ for every $\varepsilon>0$, but does not have cotype $q$. 


\section{References}

[1] C. Bennett, R. Sharpley, Interpolation of Operators, Pure Appl. Math. 129, Academic Press, Boston, 1988.

[2] J. Diestel, H. Jarchov, A. Tonge, Absolutely Summing Operators, Cambridge Stud. Adv. Math. 43, Cambridge Univ. Press, Cambridge, 1995.

[3] A. D. Ioffe, V. M. Tihomirov, Theory of Extremal Problems, Stud. Math. Appl. 6, NorthHolland, Amsterdam, 1979.

[4] N. J. Kalton, Convexity, type and the three space problem, Studia Math. 69 (1981), 247-287.

[5] N. J. Kalton, A. Kamińska, Type and order convexity of Marcinkiewicz and Lorentz spaces and applications, Glasgow Math. J. 47 (2005), 123-137.

[6] N. J. Kalton, N. T. Peck, J. W. Roberts, An F-Space Sampler, London Math. Soc. Lecture Notes Ser. 89, Cambridge Univ. Press, Cambridge, 1984.

[7] A. Kamińska, Indices, convexity and concavity in Musielak-Orlicz spaces, Funct. Approx. Comment. Math. 26 (1998), 67-84.

[8] A. Kamińska, A note on type of weak- $L^{1}$ and weak $\ell^{1}$-spaces, Banach Center Publ., this volume, 193-196.

[9] A. Kamińska, L. Maligranda, L. E. Persson, Indices and regularizations of measurable functions, in: Function Spaces (Poznań, 1998), Lecture Notes in Pure and Appl. Math. 213, Dekker, New York, 2000, 231-246.

[10] A. Kamińska, B. Turett, Type and cotype in Musielak-Orlicz spaces, in: Geometry of Banach Spaces, London Math. Soc. Lecture Note Ser. 158, Cambridge Univ. Press, Cambridge, 1990, $165-180$.

[11] M. A. Krasnoselskii, Ya. B. Rutickii, Convex Functions and Orlicz Spaces, Noordhoff, Groningen 1961.

[12] J. Lindenstrauss, L. Tzafriri, Classical Banach Spaces I, Ergeb. Math. Grenzgeb. 92, Springer, Berlin, 1977.

[13] J. Lindenstrauss, L. Tzafriri, Classical Banach Spaces II, Ergeb. Math. Grenzgeb. 97, Springer, Berlin, 1979.

[14] W. A. J. Luxemburg, Banach Function Spaces, Thesis, Delft Technical Univ., 1955.

[15] W. Matuszewska, W. Orlicz, On certain properties of $\phi$-functions, Bull. Acad. Polon. Sci. Sér. Sci. Math. Astronom. Phys. 8 (1960), 439-443.

[16] J. Musielak, Orlicz Spaces and Modular Spaces, Lecture Notes in Math. 1034, Springer, Berlin, 1983.

[17] S. Rolewicz, Metric Linear Spaces, PWN, Warszawa, 1984. 
